\title{
Modified characteristics projection finite element method for time-dependent conduction-convection problems
}

\author{
Zhiyong $\mathrm{Si}^{i^{*}}$ and Yunxia Wang ${ }^{2}$
}

${ }^{*}$ Correspondence:

sizhiyong@hpu.edu.cn

${ }^{1}$ School of Mathematics and

Information Science, Henan

Polytechnic University, Jiaozuo,

454003, P.R. China

Full list of author information is

available at the end of the article

\begin{abstract}
In this paper, we give a modified characteristics projection finite element method for the time-dependent conduction-convection problems, which is gotten by combining the modified characteristics finite element method and the projection method. The stability and the error analysis shows that our method is stable and has optimal convergence order. In order to show the effect of our method, some numerical results are presented. From the numerical results, we can see that the modified characteristics projection finite element method can simulate the fluid field, temperature field, and pressure field very well.
\end{abstract}

MSC: 76M10; 65N12; 65N30; 35K61

Keywords: time-dependent conduction-convection problems; modified characteristics method; projection method; stability analysis; error estimate

\section{Introduction}

The conduction-convection problem constitutes an important system of equations in atmospheric dynamics and dissipative nonlinear system of equations. There is a significant amount of literature as regards this problem. An optimizing reduced Petrov-Galerkin least squares mixed finite element (PLSMFE) [1] method for the non-stationary conductionconvection problems was given. An efficient sequential method was developed to estimate the unknown boundary condition on the surface of a body from transient temperature measurements inside the solid [2]. An analysis of conduction natural convection conjugate heat transfer in the gap between concentric cylinders under solar irradiation [3] was carried out by Kim et al., Boland and Layton [4] gave an error analysis for finite element methods for steady natural convection problems. Newton type iterative methods [5-7] and defect-correction methods [8-10] for the conduction-convection equations were presented.

The projection methods, which are efficient methods for solving the incompressible time-dependent fluid flow, were first introduced by Chorin [11] and Temam [12] in the late 1960s. This method is based on a special time-discretization of the Navier-Stokes equations. In this method, the convection-diffusion and the incompressibility are dealt with in two different sub-steps. The velocity obtained in the convection-diffusion sub-step is projected in order to satisfy the weak incompressibility condition. The projection methods

(c) $2015 \mathrm{Si}$ and Wang. This article is distributed under the terms of the Creative Commons Attribution 4.0 International License (http://creativecommons.org/licenses/by/4.0/), which permits unrestricted use, distribution, and reproduction in any medium, provided you give appropriate credit to the original author(s) and the source, provide a link to the Creative Commons license, and indicate if changes were made. 
can be classified into three families: the pressure-correction method [13, 14], the velocitycorrection method [15], and the consistent splitting scheme [16,17], which is called a gauge method also [18]. The convergence analysis of the semi-discrete projection methods can be found in Shen [19] and Guermond and Quartapelle [20]. In Guermond and Quartapelle [21], the projection method was implemented by the finite element method. It was used to solved the variable density Navier-Stokes equations in [22]. In [23], a gauge-Uzawa projection method was presented. Then it was applied to the conduction-convection equations [24] and incompressible flows with variable density [25].

As we know, the characteristics method is a highly effective method for the advection dominated problems. Douglas and Russell [26] presented the modified method of characteristics first. It was extended to nonlinear coupled systems by Russell [27] in two and three spatial dimensions. A detailed analysis for the Navier-Stokes equations has been done by Dawson et al. [28] and numerical tests have been presented by Buscagkia and Dari [29]. A second order MMOC mixed defect-correction finite element method [30] for time-dependent Navier-Stokes problems was given. Notsu et al. gave a single-step characteristics finite difference analysis for the convection-diffusion problems [31] and a single-step finite element method for the incompressible Navier-Stokes equations [32]. El-Amrani and Seaid gave the error estimates of the modified method of characteristics finite element methods for the Navier-Stokes [33], natural, and mixed convection flows [34]. In [35], Achdou and Guermond gave the projection/Lagrange-Galerkin method for the incompressible Navier-Stokes equations.

In this paper, we give the modified characteristics projection finite element method (MCPFEM) for the time-dependent conduction-convection problems, which is gotten by combining the modified characteristics finite element method and the projection method. We give stability and error analysis, which show that our method is stable and has optimal convergence order. In order to show the efficiency of our method, some numerical results are presented. At first, the numerical results of Bénard convection problems are given. Then we give some numerical results of the thermal driven cavity flow. From the numerical results, we can see that MCPFEM can simulate the fluid field, temperature field, and pressure field very well.

\section{The modified characteristics projection finite element method for the time-dependent conduction-convection problems}

In this paper, we consider the time-dependent conduction-convection problem in two dimensions whose coupled equations governing viscous incompressible flow and heat transfer for the incompressible fluid are Boussinesq approximations to the Navier-Stokes equations. For all $t \in\left(0, t_{1}\right]$, find $(u, p, T) \in X \times M \times W$ such that

$$
\begin{cases}u_{t}-v \Delta u+(u \cdot \nabla) u+\nabla p=\kappa v^{2} g T+f, & x \in \Omega, \\ \operatorname{div} u=0, & x \in \Omega, \\ T_{t}-\lambda v \Delta T+u \cdot \nabla T=b, & x \in \Omega, \\ u(x, 0)=u_{0}, \quad T(x, 0)=T_{0}, & x \in \Omega, \\ u=0, \quad T=0, & x \in \partial \Omega,\end{cases}
$$

where $\Omega$ is a bounded domain in $\mathbb{R}^{2}$ assumed to have a Lipschitz continuous boundary $\partial \Omega . u=\left(u_{1}(x, t), u_{2}(x, t)\right)^{T}$ represents the velocity vector, $p(x, t)$ represents the pressure, 
$T(x, t)$ represents the temperature, $\kappa$ represents the Grashoff number, $\lambda=\operatorname{Pr}^{-1}, \operatorname{Pr}$ is the Prandtl number, $g$ represents the vector of gravitational acceleration, $v=R e^{-1}, R e$ is the Reynolds number, and $f$ and $b$ are the forcing functions.

In this section, we aim to describe some notations and materials which will be frequently used in this paper. For the mathematical setting of the conduction-convection problems (1), we introduce the Hilbert spaces

$$
\begin{aligned}
& X=H_{0}^{1}(\Omega)^{2}, \quad W=H^{1}(\Omega) \\
& M=L_{0}^{2}(\Omega)=\left\{\varphi \in L^{2}(\Omega) ; \int_{\Omega} \varphi d x=0\right\}
\end{aligned}
$$

$\Im_{h}$ is a quasi-uniform partition of $\bar{\Omega}_{h}$ into non-overlapping triangles, indexed by a parameter $h=\max _{K \in \Im_{h}}\left\{h_{K} ; h_{K}=\operatorname{diam}(K)\right\}$. We introduce the finite element subspace $X_{h} \subset X$, $M_{h} \subset M, W_{h} \subset W$ as follows:

$$
\begin{aligned}
& X_{h}=\left\{v_{h} \in X \cap C^{0}(\bar{\Omega})^{2} ;\left.v_{h}\right|_{K} \in P_{\ell}(K)^{2}, \forall K \in \Im_{h}\right\}, \\
& M_{h}=\left\{q_{h} \in M \cap C^{0}(\bar{\Omega}) ;\left.q_{h}\right|_{K} \in P_{k}(K), \forall K \in \Im_{h}\right\}, \\
& W_{h}=\left\{\phi_{h} \in W \cap C^{0}(\bar{\Omega}) ;\left.\phi_{h}\right|_{K} \in P_{j}(K), \forall K \in \Im_{h}\right\},
\end{aligned}
$$

where $P_{\ell}(K)$ is the space of piecewise polynomials of degree $\ell$ on $K, \ell \geq 1, k \geq 1, j \geq 1$ are three integers. $W_{0 h}=W_{h} \cap H_{0}^{1}(\Omega)$, and assume that $\left(X_{h}, M_{h}\right)$ satisfies the discrete LBB condition, there exists $\beta>0$ such that

$$
\sup _{v_{h} \in X_{h}} \frac{d\left(v_{h}, \varphi_{h}\right)}{\left\|\nabla v_{h}\right\|_{0}} \geq \beta\left\|\varphi_{h}\right\|_{0}, \quad \forall \varphi_{h} \in M_{h},
$$

where $d\left(v_{h}, \varphi_{h}\right)=-\left(\varphi_{h}, \nabla \cdot v_{h}\right)$. Let $V_{h}$ be the kernel of the discrete divergence operator,

$$
V_{h}=\left\{v_{h} \in X_{h} ;\left(q_{h}, \nabla \cdot v_{h}\right)=0, \forall q_{h} \in M_{h}\right\}
$$

For each positive integer $N$, let $\left\{\mathcal{J}_{n}: 1 \leq n \leq N\right\}$ be a partition of $\left[0, t_{1}\right]$ into subintervals $\mathcal{J}_{n}=\left(t_{n-1}, t_{n}\right]$, with $t_{n}=n \tau, \tau=T_{1} / N$. Set $u^{n}=u\left(\cdot, t_{n}\right)$. The characteristic trace-back along the field $u^{n-1}$ of a point $x \in \Omega$ at time $t_{n}$ to $t_{n-1}$ is approximately

$$
\bar{x}\left(x, t_{n-1}\right)=x-u^{n-1} \tau .
$$

Consequently, the hyperbolic part in the first equation of (1) at time $t_{n}$ is approximated by

$$
u_{t}+u^{n-1} \cdot \nabla u^{n} \approx \frac{u^{n}-\bar{u}^{n-1}}{\Delta t}
$$

where

$$
\bar{u}^{n-1}= \begin{cases}u^{n-1}(\bar{x}), & \bar{x}=x-u^{n-1} \tau \in \Omega, \\ 0, & \text { otherwise }\end{cases}
$$

for any function $w$. 
With the previous notations, we get the projection FEM for the time-dependent conduction-convection problem (1), which is a slight transmutation of the projection FEM $[13,19]$ for the time-dependent Navier-Stokes equations.

Algorithm 2.1 (Projection FEM) Start $u_{h}^{0}$ as a solution of $\left(u_{h}^{0}, v_{h}\right)=\left(u_{0}, v_{h}\right)$ and $\left(T_{h}^{0}, \psi_{h}\right)=$ $\left(T_{0}, \psi_{h}\right), p_{h}^{0}=0$ for all $v_{h} \in V_{h}, \psi_{h} \in M_{h}$.

Step 1: Find $\hat{u}_{h}^{n+1} \in X_{h}$ as the solution of

$$
\begin{gathered}
\left(\frac{\hat{u}_{h}^{n+1}-u_{h}^{n}}{\tau}, v_{h}\right)+B\left(u_{h}^{n}, \hat{u}_{h}^{n+1}, v_{h}\right)+v\left(\nabla \hat{u}_{h}^{n+1}, \nabla v_{h}\right) \\
=\kappa v^{2}\left(g T_{h}^{n}, v_{h}\right)+\left(f\left(t_{n+1}\right), v_{h}\right), \quad \forall v_{h} \in X_{h},
\end{gathered}
$$

where $B\left(u_{h}, v_{h}, w_{h}\right)=\frac{1}{2}\left(\left(u_{h} \cdot \nabla\right) v_{h}, w_{h}\right)-\frac{1}{2}\left(\left(u_{h} \cdot \nabla\right) w_{h}, v_{h}\right)$.

Step 2: Find $u_{h}^{n+1} \in V_{h}, p_{h}^{n+1} \in M_{h}$ as the solution of

$$
\begin{aligned}
& \left(\frac{u_{h}^{n+1}-\hat{u}_{h}^{n+1}}{\tau}, v_{h}\right)+d\left(p_{h}^{n+1}, v_{h}\right)=0, \quad \forall v_{h} \in V_{h}, \\
& d\left(q_{h}, u_{h}^{n+1}\right)=0, \quad \forall q_{h} \in M_{h} .
\end{aligned}
$$

Step 3: Compute $T_{h}^{n+1} \in W_{h}$ as the solution of the linear elliptic equation

$$
\begin{gathered}
\left(\frac{T_{h}^{n+1}-T_{h}^{n}}{\tau}, \psi_{h}\right)+\bar{B}\left(u_{h}^{n+1}, T_{h}^{n+1}, \psi_{h}\right)+\lambda v\left(\nabla T_{h}^{n+1}, \nabla \psi_{h}\right) \\
=\left(b\left(t_{n+1}\right), \psi_{h}\right), \quad \forall \psi_{h} \in W_{0 h}, \\
\bar{B}\left(u_{h}, T_{h}, \psi_{h}\right)=\frac{1}{2}\left(\left(u_{h} \cdot \nabla\right) T_{h}, \psi_{h}\right)-\frac{1}{2}\left(\left(u_{h} \cdot \nabla\right) \psi_{h}, T_{h}\right) .
\end{gathered}
$$

Remark 2.1 Denote by $P_{h}$ the orthogonal projector in $\left(L^{2}(\Omega)\right)^{2}$ onto $V$. We can readily check that (2) is equivalent to [19]

$$
u_{h}^{n+1}=P_{h} \hat{u}_{h}^{n+1} .
$$

The MC time discretization, combined with the projection finite element method, leads to the following MC projection finite element method.

Algorithm 2.2 (MC projection FEM) Start with $u_{h}^{0}$ as a solution of $\left(u_{h}^{0}, v_{h}\right)=\left(u_{0}, v_{h}\right)$ for all $v_{h} \in V_{h}$.

Step 1: Find $\hat{u}_{h}^{n+1} \in X_{h}$ as the solution of

$$
\begin{aligned}
& \left(\frac{\hat{u}_{h}^{n+1}-\dot{u}_{h}^{n}}{\tau}, v_{h}\right)+v\left(\nabla \hat{u}_{h}^{n+1}, \nabla v_{h}\right) \\
& =\kappa v^{2}\left(g T_{h}^{n}, v_{h}\right)+\left(f\left(t_{h+1}\right), v_{h}\right), \quad \forall v_{h} \in V_{h},
\end{aligned}
$$

where

$$
\dot{u}_{h}^{n}= \begin{cases}u_{h}^{n}(\dot{x}), & \dot{x}=x-u_{h}^{n} \tau \in \Omega, \\ 0, & \text { otherwise }\end{cases}
$$


Step 2: Find $u_{h}^{n+1} \in V_{h}, p_{h}^{n+1} \in M_{h}$ as the solution of

$$
\begin{aligned}
& \left(\frac{u_{h}^{n+1}-\hat{u}_{h}^{n+1}}{\tau}, v_{h}\right)+b\left(p_{h}^{n+1}, v_{h}\right)=0, \quad \forall v_{h} \in V_{h}, \\
& b\left(q_{h}, u_{h}^{n+1}\right)=0, \quad \forall q_{h} \in M_{h} .
\end{aligned}
$$

Step 3: Compute $T_{h}^{n+1} \in W_{h}$, the solution of the linear elliptic equation

$$
\left(\frac{T_{h}^{n+1}-\dot{T}_{h}^{n}}{\tau}, \psi_{h}\right)+\lambda \nu\left(\nabla T_{h}^{n+1}, \nabla \psi_{h}\right)=\left(b\left(t_{n+1}\right), \psi_{h}\right), \quad \forall \psi_{h} \in W_{0 h},
$$

where

$$
\dot{T}_{h}^{n}= \begin{cases}T_{h}^{n}(\dot{x}), & \dot{x}=x-u_{h}^{n} \tau \in \Omega \\ 0, & \text { otherwise. }\end{cases}
$$

Remark 2.2 Define $\dot{\mathcal{X}}_{x}^{n+1}(t)=x-\left(t_{n+1}-t\right) u_{h}^{n}, \forall t \in\left[t_{n-1}, t_{n+1}\right], 2 \leq l \leq N$. Since $X_{h}$ is a subset of $W^{1, \infty}(\Omega)$, under the condition $\tau \leq \frac{1}{2 L_{n}}, L_{n}=\max _{1 \leq i \leq n}\left\|u_{h}^{n}\right\|_{W^{1, \infty}}$ on the time step it is an easy matter to verify that this mapping has a positive Jacobian, since $u_{h}^{n}$ vanishes on $\partial \Omega$; this mapping is one-to-one and this is a change of variables from $\Omega$ onto $\Omega$. This yields for any positive function $\phi$ on $\Omega$ the estimate (please see [36] for details)

$$
\int_{\Omega} \phi\left(\dot{\mathcal{X}}_{h}^{n+1}(t)\right) d x \leq C \int_{\Omega} \phi(x) d x
$$

\section{Stability analysis}

Theorem 3.1 (Stability) If $\tau \leq \frac{1}{2 L_{n}}, L_{n}=\max _{1 \leq i \leq n}\left\|u_{h}^{i}\right\|_{W^{1, \infty}}$, the MC projection FEM is stable in the sense that

$$
\begin{aligned}
& \left\|u_{h}^{N+1}\right\|_{0}^{2}+\left\|T_{h}^{N+1}\right\|_{0}^{2}+2 \nu \tau \sum_{n=1}^{N}\left\|\nabla u_{h}^{n+1}\right\|_{0}^{2}+\lambda \nu \tau \sum_{n=1}^{N}\left\|\nabla T_{h}^{n+1}\right\|_{0}^{2} \\
& \leq C\left\|u_{h}^{0}\right\|_{0}^{2}+C\left\|T_{h}^{0}\right\|_{0}^{2}+C \frac{\tau}{2 v} \sum_{n=1}^{N}\left\|f\left(t_{n+1}\right)\right\|_{-1}^{2}+2 C \tau \sum_{n=1}^{N}\left\|b\left(t_{n+1}\right)\right\|_{-1} .
\end{aligned}
$$

Remark 3.1 We will prove the boundary of $\left\|u_{h}^{n}\right\|_{W^{1, \infty}}$ in the next section. Here, we use mathematical induction method.

Proof Let $v_{h}=u_{h}^{n+1}$ in (5), we obtain

$$
\left(\frac{\hat{u}_{h}^{n+1}-\dot{u}_{h}^{n}}{\tau}, u_{h}^{n+1}\right)+v\left(\nabla \hat{u}_{h}^{n+1}, \nabla u_{h}^{n+1}\right)=\kappa v^{2}\left(g T_{h}^{n}, u_{h}^{n+1}\right)+\left(f\left(t_{n+1}\right), u_{h}^{n+1}\right) .
$$

Using (6), we deduce

$$
\left(\hat{u}_{h}^{n+1}, v_{h}\right)=\left(u_{h}^{n+1}, v_{h}\right)+\tau d\left(p_{h}, v_{h}\right), \quad \forall v_{h} \in X_{h}
$$

Noting $\nabla \cdot u_{h}^{n+1}=0$, we get

$$
\left(\hat{u}_{h}^{n+1}, u_{h}^{n+1}\right)=\left(u_{h}^{n+1}, u_{h}^{n+1}\right),
$$




$$
\left(\nabla \hat{u}_{h}^{n+1}, \nabla u_{h}^{n+1}\right)=\left(\nabla u_{h}^{n+1}, \nabla u_{h}^{n+1}\right)
$$

Then we deduce

$$
\left(\frac{u_{h}^{n+1}-\dot{u}_{h}^{n}}{\tau}, u_{h}^{n+1}\right)+v\left\|\nabla u_{h}^{n+1}\right\|_{0}^{2}=\kappa v^{2}\left(g T_{h}^{n}, u_{h}^{n+1}\right)+\left(f\left(t_{n+1}\right), u_{h}^{n+1}\right) .
$$

We arrive at

$$
\begin{aligned}
& \left\|u_{h}^{n+1}\right\|_{0}^{2}-\left\|u_{h}^{n}\right\|_{0}^{2}+2 \nu \tau\left\|\nabla \hat{u}_{h}^{n+1}\right\|_{0}^{2} \\
& \quad \leq\left\|\dot{u}_{h}^{n}\right\|_{0}^{2}-\left\|u_{h}^{n}\right\|_{0}^{2}+2 \kappa v^{2} \tau\left(g T_{h}^{n}, \hat{u}_{h}^{n+1}\right)+2 \tau\left(f\left(t_{n+1}\right), \hat{u}_{h}^{n+1}\right) .
\end{aligned}
$$

Now, we estimate the bound of $\left\|\dot{u}_{h}^{n}\right\|_{0}^{2}-\left\|u_{h}^{n}\right\|_{0}^{2}$. By the definition of $\dot{\mathcal{X}}_{x}^{n}\left(t_{n-1}\right)$, we have

$$
J\left(\dot{\mathcal{X}}_{x}^{n}\left(t_{n-1}\right)\right)=\left(\begin{array}{cc}
1-\partial_{x} u_{h 1}^{n-1} \tau & -\partial_{y} u_{h 1}^{n-1} \tau \\
-\partial_{x} u_{h 2}^{n-1} \tau & 1-\partial_{y} u_{h 2}^{n-1} \tau
\end{array}\right)
$$

Hence,

$$
\operatorname{det} J\left(\dot{\mathcal{X}}_{x}^{n}\left(t_{n-1}\right)\right)=1+\mathcal{O}(\tau)
$$

Then we get

$$
\begin{aligned}
\left\|\dot{u}_{h}^{n}\right\|_{0}^{2}-\left\|u_{h}^{n}\right\|_{0}^{2} & =\int_{\Omega}\left(\dot{u}_{h}^{n}\right)^{2} d x-\int_{\Omega}\left(u_{h}^{n}\right)^{2} d x \\
& =\int_{\Omega}\left(u_{h}^{n}\right)^{2}(1+\mathcal{O}(\tau)) d x-\int_{\Omega}\left(u_{h}^{n}\right)^{2} d x .
\end{aligned}
$$

We have

$$
\left\|\dot{u}_{h}^{n}\right\|_{0}^{2}-\left\|u_{h}^{n}\right\|_{0}^{2} \leq C \tau\left\|u_{h}^{n}\right\|_{0}^{2}
$$

On the other hand, by Cauchy-Schwarz inequality, we deduce

$$
\begin{aligned}
2 \kappa \nu^{2} \tau\left(g T_{h}^{n}, u_{h}^{n+1}\right) & \leq 2 C \kappa \nu^{2} \tau\left\|T_{h}^{n}\right\|_{0}\left\|u_{h}^{n+1}\right\|_{0} \\
& \leq C \kappa^{2} \nu^{4} \tau\left\|T_{h}^{n}\right\|_{0}^{2}+C \tau\left\|u_{h}^{n+1}\right\|_{0}^{2} .
\end{aligned}
$$

Combining (10), (11), and (12), we get

$$
\begin{aligned}
& \left\|u_{h}^{n+1}\right\|_{0}^{2}-\left\|u_{h}^{n}\right\|_{0}^{2}+2 v \tau\left\|\nabla u_{h}^{n+1}\right\|_{0}^{2} \\
& \leq C \tau\left\|u_{h}^{n}\right\|_{0}^{2}+\frac{\tau}{2 v}\left\|f\left(t_{n+1}\right)\right\|_{-1}^{2}+\frac{3 v \tau}{2}\left\|\nabla u_{h}^{n+1}\right\|_{0}^{2} \\
& \quad+C \kappa^{2} v^{4} \tau\left\|T_{h}^{n}\right\|_{0}^{2}+C \tau\left\|u_{h}^{n+1}\right\|_{0}^{2} .
\end{aligned}
$$

Let $\psi_{h}=2 \tau T_{h}^{n+1}$ in (7), we obtain

$$
2\left(T_{h}^{n+1}-\dot{T}_{h}^{n}, T_{h}^{n+1}\right)+2 \tau \lambda \nu\left(\nabla T_{h}^{n+1}, \nabla T_{h}^{n+1}\right)=2 \tau\left(b\left(t_{n+1}\right), T_{h}^{n+1}\right) .
$$


We deduce

$$
\left\|T_{h}^{n+1}\right\|_{0}^{2}-\left\|T_{h}^{n}\right\|_{0}^{2}+\tau \lambda \nu\left\|\nabla T_{h}^{n+1}\right\|_{0}^{2} \leq 2 \tau\left\|b\left(t_{n+1}\right)\right\|_{-1}+\left\|\dot{T}_{h}^{n}\right\|_{0}^{2}-\left\|T_{h}^{n}\right\|_{0}^{2} .
$$

Similar to (11), we have

$$
\left\|\dot{T}_{h}^{n}\right\|_{0}^{2}-\left\|T_{h}^{n}\right\|_{0}^{2} \leq C \tau\left\|u_{h}^{n}\right\|_{0}^{2} .
$$

Then we can get

$$
\left\|T_{h}^{n+1}\right\|_{0}^{2}-\left\|T_{h}^{n}\right\|_{0}^{2}+\tau \lambda \nu\left\|\nabla T_{h}^{n+1}\right\|_{0}^{2} \leq 2 \tau\left\|b\left(t_{n+1}\right)\right\|_{-1}+C \tau\left\|u_{h}^{n}\right\|_{0}^{2} .
$$

Adding (13) and (15), summing over all $n$ from 0 to $N$, we can get

$$
\begin{aligned}
& \left\|u_{h}^{N+1}\right\|_{0}^{2}+\left\|T_{h}^{N+1}\right\|_{0}^{2}+2 v \tau \sum_{n=1}^{N}\left\|\nabla u_{h}^{n+1}\right\|_{0}^{2}+\lambda \nu \tau \sum_{n=1}^{N}\left\|\nabla T_{h}^{n+1}\right\|_{0}^{2} \\
& \leq\left\|u_{h}^{0}\right\|_{0}^{2}+\left\|T_{h}^{0}\right\|_{0}^{2}+C \tau \sum_{n=1}^{N}\left\|u_{h}^{n+1}\right\|_{0}^{2} \\
& \quad+\frac{\tau}{2 v} \sum_{n=1}^{N}\left\|f\left(t_{n+1}\right)\right\|_{-1}^{2}+C \kappa^{2} \nu^{4} \tau \sum_{n=1}^{N}\left\|T_{h}^{n}\right\|_{0}^{2}+2 \tau \sum_{n=1}^{N}\left\|b\left(t_{n+1}\right)\right\|_{-1} .
\end{aligned}
$$

Using Gronwall lemma, we deduce

$$
\begin{aligned}
& \left\|u_{h}^{N+1}\right\|_{0}^{2}+\left\|T_{h}^{N+1}\right\|_{0}^{2}+2 \nu \tau \sum_{n=1}^{N}\left\|\nabla u_{h}^{n+1}\right\|_{0}^{2}+\lambda \nu \tau \sum_{n=1}^{N}\left\|\nabla T_{h}^{n+1}\right\|_{0}^{2} \\
& \leq C\left\|u_{h}^{0}\right\|_{0}^{2}+C\left\|T_{h}^{0}\right\|_{0}^{2}+C \frac{\tau}{2 v} \sum_{n=1}^{N}\left\|f\left(t_{n+1}\right)\right\|_{-1}^{2}+2 C \tau \sum_{n=1}^{N}\left\|b\left(t_{n+1}\right)\right\|_{-1} .
\end{aligned}
$$

\section{Error analysis}

In order to get the error analysis, we give some lemmas first.

Lemma 4.1 $[37,38]$ Let $e(x, n)=\left[\frac{u^{n}(x)-\bar{u}^{n-1}(x)}{\tau}-\left(\frac{\partial u}{\partial t}\left(x, t_{n}\right)+u^{n}(x) \nabla u^{n}(x)\right)\right]$ and let $\tau>0$ be such that $u \in \mathscr{C}^{4}\left([\tau, T] ; H^{3}(\Omega)^{2}\right)$. For $t_{n}>\tau$, we have

$$
e(x, n)=-\tau\left(\frac{1}{2} \frac{d^{2} g_{x}^{n}}{d t^{2}}+\frac{\partial u}{\partial t} \cdot \nabla u\left(x, t_{n}\right)\right)+O\left(\tau^{2}\right)
$$

where $g_{x}^{n}(t)=u\left(x-\left(t_{n}-t\right) u^{n-1}, t\right), u^{n}(x)=u\left(x, t_{n}\right)$.

Lemma 4.2 Let

$$
\zeta(x, n)=\left(\frac{T^{n}(x)-\dot{T}^{n-1}(x)}{\tau}-T_{t}(x, t)-u \cdot \nabla T\right),
$$

and let $\tau>0$ be such that $T \in \mathscr{C}^{4}\left([\tau, T] ; H^{3}(\Omega)\right)$. For $t_{n}>\tau$, we have

$$
\zeta(x, n)=-\tau\left(\frac{1}{2} \frac{d^{2} \gamma_{x}^{n}}{d t^{2}}+\frac{\partial u}{\partial t} \cdot \nabla T\left(x, t_{n}\right)\right)+O\left(\tau^{2}\right),
$$

where $\gamma_{x}^{n}(t)=T\left(x-\left(t_{n}-t\right) u_{h}^{n-1}, t\right), u^{n}(x)=u\left(x, t_{n}\right)$. 
Lemma 4.3 There exists $r_{h}: W \rightarrow W_{h}$; for all $\psi \in W$ we have

$$
\begin{aligned}
& \left(\nabla\left(\psi-r_{h} \psi\right), \phi_{h}\right)=0, \quad \forall \phi_{h} \in W_{h}, \\
& \int_{\Omega}\left(\psi-r_{h} \psi\right) d x=0, \quad\left\|\nabla r_{h} \psi\right\|_{0} \leq\|\nabla \psi\|_{0} .
\end{aligned}
$$

When $\psi \in W^{r, q}(\Omega)(1 \leq q \leq \infty)$, we have

$$
\left\|\psi-r_{h} \psi\right\|_{-s, q} \leq C h^{r+s}|\psi|_{r, q}, \quad-1 \leq s \leq m, 0 \leq r \leq m+1 .
$$

There exists $\bar{r}_{h}: W_{0} \rightarrow W_{0 h}$; for all $\psi \in W_{0}$ we have

$$
\left(\nabla\left(\psi-\bar{r}_{h} \psi\right), \phi_{h}\right)=0, \quad \forall \phi_{h} \in W_{0 h},\left\|\nabla \bar{r}_{h} \psi\right\|_{0} \leq\|\nabla \psi\|_{0} .
$$

When $\psi \in W^{r, q}(\Omega)(1 \leq q \leq \infty)$, we have

$$
\left\|\psi-\bar{r}_{h} \psi\right\|_{-s, q} \leq C h^{r+s}|\psi|_{r, q}, \quad-1 \leq s \leq m, 0 \leq r \leq m+1 .
$$

Then we define the Galerkin projection $\left(R_{h}, Q_{h}\right)=\left(R_{h}(u, p), Q_{h}(u, p)\right):(X, M) \rightarrow\left(X_{h}\right.$, $M_{h}$ ), such that

$$
\begin{gathered}
v a\left(R_{h}-u, v_{h}\right)-d\left(Q_{h}-p, v_{h}\right)+d\left(\varphi_{h}, R_{h}-u\right)=0, \\
\forall(u, p) \in(X, M),\left(v_{h}, \varphi_{h}\right) \in\left(X_{h}, M_{h}\right) .
\end{gathered}
$$

Lemma 4.4 $[39,40]$ The Galerkin projection $\left(R_{h}, Q_{h}\right)$ satisfies

$$
\begin{aligned}
& \left\|R_{h}-u\right\|_{0}+h\left(\left\|\nabla\left(R_{h}-u\right)\right\|_{0}+\left\|Q_{h}-p\right\|_{0}\right) \leq C h^{k+1}\left(v\|u\|_{k+1}+\|p\|_{k}\right), \\
& \quad k=1,2 .
\end{aligned}
$$

\subsection{Error estimate for velocity and temperature}

Lemma 4.5 If $\tau \leq \frac{1}{2 L_{n}}, L_{n}=\max _{1 \leq i \leq n}\left\|u_{h}^{i}\right\|_{W^{1, \infty}}, u, p, u_{t}$, and $p_{t}$ are sufficiently smooth, we have

$$
\begin{aligned}
& \left\|u_{h}^{n+1}\right\|_{W^{1, \infty}}<+\infty, \\
& \left\|\xi_{h}^{N+1}\right\|_{0}^{2}+\sum_{n=0}^{N}\left\|\hat{\xi}_{h}^{n+1}-\xi_{h}^{n}\right\|_{0}^{2}+\frac{1}{2} \nu \tau \sum_{n=0}^{N}\left\|\nabla \hat{\xi}_{h}^{n+1}\right\|_{0}^{2}+\left\|\varepsilon_{h}^{N+1}\right\|_{0}^{2} \\
& \quad+\sum_{n=0}^{N}\left\|\varepsilon_{h}^{n+1}-\xi_{h}^{n}\right\|_{0}^{2}+\lambda \nu \tau \sum_{n=0}^{N}\left\|\nabla \varepsilon_{h}^{n+1}\right\|_{0}^{2} \leq C\left(\tau^{2}+h^{2(k+1)}\right), \\
& \left\|\xi_{h}^{N+1}\right\|_{0}^{2}+\sum_{n=0}^{N}\left\|\hat{\xi}_{h}^{n+1}-\xi_{h}^{n}\right\|_{0}^{2}+\frac{1}{2} \nu \tau \sum_{n=0}^{N}\left\|\nabla \xi_{h}^{n+1}\right\|_{0}^{2}+\left\|\varepsilon_{h}^{N+1}\right\|_{0}^{2} \\
& \quad+\sum_{n=0}^{N}\left\|\varepsilon_{h}^{n+1}-\xi_{h}^{n}\right\|_{0}^{2}+\lambda \nu \tau \sum_{n=0}^{N}\left\|\nabla \varepsilon_{h}^{n+1}\right\|_{0}^{2} \leq C\left(\tau^{2}+h^{2(k+1)}\right),
\end{aligned}
$$


where $\hat{\xi}_{h}^{n}=\hat{u}_{h}^{n}-R_{h}^{n}, \xi_{h}^{n}=u_{h}^{n}-R_{h}^{n}, \varepsilon_{h}^{n+1}=T_{h}^{n+1}-r_{h} T^{n+1}, R_{h}^{n}=R_{h}\left(u^{n}, p^{n}\right), C$ is a positive constant independent of $\tau$ and $h$.

Proof Subtracting $\left(\frac{R_{h}^{n+1}-\dot{R}_{h}^{n}}{\tau}, v_{h}\right)+v\left(\nabla R_{h}^{n+1}, \nabla v_{h}\right)$ from both sides of (5), we can get

$$
\begin{aligned}
& \left(\frac{\left(\hat{u}_{h}^{n+1}-R_{h}^{n+1}\right)-\left(\dot{u}_{h}^{n}-\dot{R}_{h}^{n}\right)}{\tau}, v_{h}\right)+v\left(\nabla\left(\hat{u}_{h}^{n+1}-R_{h}^{n+1}\right), \nabla v_{h}\right) \\
& \quad=k v^{2} g\left(T_{h}^{n}, v_{h}\right)+\left(f^{n}, v_{h}\right)-\left(\frac{R_{h}^{n+1}-\dot{R}_{h}^{n}}{\tau}, v_{h}\right)-v\left(\nabla R_{h}^{n+1}, \nabla v_{h}\right) .
\end{aligned}
$$

Defining $\eta^{n}=u^{n}-R_{h}^{n}$, we can get

$$
\begin{aligned}
&\left(\frac{\hat{\xi}_{h}^{n+1}-\xi_{h}^{n}}{\tau}, v_{h}\right)+v\left(\nabla \hat{\xi}_{h}^{n+1}, \nabla v_{h}\right) \\
&=-\left(\frac{u^{n+1}-\bar{u}^{n}}{\tau}-v \Delta u^{n+1}+\nabla p^{n+1}-k v^{2} g T^{n+1}-f^{n+1}, v_{h}\right) \\
&+\left(\frac{\eta^{n+1}-\dot{\eta}^{n}}{\tau}, v_{h}\right)+\left(\frac{\dot{u}^{n}-\bar{u}^{n}}{\tau}, v_{h}\right)+\left(\nabla p^{n+1}, v_{h}\right)+\left(\frac{\dot{\xi}_{h}^{n}-\xi_{h}^{n}}{\tau}, v_{h}\right) \\
&+v\left(\nabla\left(u^{n+1}-R_{h}^{n+1}\right), \nabla v_{h}\right)+k v^{2} g\left(T^{n+1}-T_{h}^{n}, v_{h}\right) \\
&=-\left(\frac{u^{n+1}-\bar{u}^{n}}{\tau}-v \Delta u^{n+1}+\nabla p^{n+1}-k v^{2} g T^{n+1}-f^{n+1}, v_{h}\right) \\
&+\left(\frac{\dot{u}^{n}-\bar{u}^{n}}{\tau}, v_{h}\right)+d\left(Q_{h}^{n+1}-p^{n+1}, v_{h}\right)+\left(\frac{\eta^{n+1}-\dot{\eta}^{n}}{\tau}, v_{h}\right) \\
&+\left(\frac{\dot{\xi}_{h}^{n}-\xi_{h}^{n}}{\tau}, v_{h}\right)+v\left(\nabla\left(u^{n+1}-R_{h}^{n+1}\right), \nabla v_{h}\right)+k v^{2} g\left(T^{n+1}-T_{h}^{n}, v_{h}\right) \\
&=\left(\frac{u^{n+1}-\bar{u}^{n}}{\tau}-v \Delta u^{n+1}+\nabla p^{n+1}-k v^{2} g T^{n+1}-f^{n+1}, v_{h}\right) \\
&+\left(\frac{\eta^{n+1}-\dot{\eta}^{n}}{\tau}, v_{h}\right)+\left(\frac{\dot{u}^{n}-\bar{u}^{n}}{\tau}, v_{h}\right)+\left(\frac{\dot{\xi}_{h}^{n}-\xi_{h}^{n}}{\tau}, v_{h}\right) \\
&+k v^{2} g\left(T^{n+1}-T_{h}^{n}, v_{h}\right) .
\end{aligned}
$$

Let $v_{h}=2 \tau \hat{\xi}_{h}^{n+1}$ in (26), we can get

$$
\begin{aligned}
\left\|\hat{\xi}_{h}^{n+1}\right\|_{0}^{2}-\left\|\xi_{h}^{n}\right\|_{0}^{2}+\left\|\hat{\xi}_{h}^{n+1}-\xi_{h}^{n}\right\|_{0}^{2}+2 v \tau\left\|\nabla \hat{\xi}_{h}^{n+1}\right\|_{0}^{2} \\
=-2 \tau\left(\frac{u^{n+1}-\bar{u}^{n}}{\tau}-v \Delta u^{n+1}+\nabla p^{n+1}-k v^{2} g T^{n+1}-f^{n+1}, \hat{\xi}_{h}^{n+1}\right) \\
\quad+2\left(\eta^{n+1}-\dot{\eta}^{n}, \hat{\xi}_{h}^{n+1}\right)+2\left(\dot{u}^{n}-\bar{u}^{n}, \hat{\xi}_{h}^{n+1}\right)+2\left(\dot{\xi}_{h}^{n}-\xi_{h}^{n}, \hat{\xi}_{h}^{n+1}\right) \\
\quad+k v^{2} \tau g\left(T^{n+1}-T_{h}^{n}, \hat{\xi}_{h}^{n+1}\right) \\
\equiv \sum_{i=1}^{5} \mathcal{A}_{i},
\end{aligned}
$$


where

$$
\begin{aligned}
& \mathcal{A}_{1}=-2 \tau\left(\frac{u^{n+1}-\bar{u}^{n}}{\tau}-v \Delta u^{n+1}+\nabla p^{n+1}-k v^{2} g T^{n+1}-f^{n+1}-f^{n+1}, \hat{\xi}_{h}^{n+1}\right), \\
& \mathcal{A}_{2}=2\left(\dot{u}^{n}-\bar{u}^{n}, \hat{\xi}_{h}^{n+1}\right) \\
& \mathcal{A}_{3}=2\left(\eta^{n+1}-\dot{\eta}^{n}, \hat{\xi}_{h}^{n+1}\right) \\
& \mathcal{A}_{4}=2\left(\dot{u}^{n}-\bar{u}^{n}, \hat{\xi}_{h}^{n+1}\right)+2\left(\dot{\xi}_{h}^{n}-\xi_{h}^{n}, \hat{\xi}_{h}^{n+1}\right), \\
& \mathcal{A}_{5}=k v^{2} g \tau\left(T^{n+1}-T_{h}^{n}, \hat{\xi}_{h}^{n+1}\right) .
\end{aligned}
$$

Now, we estimate each term $\mathcal{A}_{i}$, respectively. By Hölder inequality, we get

$$
\mathcal{A}_{1} \leq C \tau\left\|\bar{e}^{n+1}\right\|_{0}^{2}+\frac{\nu \tau}{8}\left\|\nabla \hat{\xi}_{h}^{n+1}\right\|_{0}^{2}
$$

By the definition of $\dot{x}$ and $\bar{x}$, we can get

$$
\dot{x}\left(x, t_{n}\right)-\bar{x}\left(x, t_{n}\right)=\left(u_{h}^{n}-u^{n}\right) \tau .
$$

Using Taylor's formula, we obtain

$$
\begin{aligned}
\left|\dot{u}^{n}-\bar{u}^{n}\right| & =\left|u^{n}(\dot{x})-u^{n}(\bar{x})\right| \\
& \leq \tau\left\|\nabla u^{n}\right\|_{\infty}\left|u_{h}^{n}-u^{n}\right| \\
& \leq \tau\left\|\nabla u^{n}\right\|_{\infty}\left(\left|u_{h}^{n}-R_{h}^{n}\right|+\left|R_{h}^{n}-u^{n}\right|\right) .
\end{aligned}
$$

Therefore, we have

$$
\begin{aligned}
\left\|\dot{u}^{n}-\bar{u}^{n}\right\|_{0} & \leq \tau\left\|\nabla u^{n}\right\|_{\infty}\left(\left\|u^{n}-R_{h}^{n}\right\|_{0}+\left\|R_{h}^{n}-u_{h}^{n}\right\|_{0}\right) \\
& \leq C \tau\left(h^{k+1}+\left\|\xi_{h}^{n}\right\|_{0}\right) .
\end{aligned}
$$

Then we deduce

$$
\begin{aligned}
\mathcal{A}_{2} & \leq C\left\|\dot{u}^{n}-\bar{u}^{n}\right\|_{0}\left\|\nabla \hat{\xi}_{h}^{n+1}\right\|_{0} \\
& \leq C \tau\left(h^{2(k+1)}+\left\|\xi_{h}^{n}\right\|_{0}^{2}\right)+\frac{\nu \tau}{8}\left\|\nabla \hat{\xi}_{h}^{n+1}\right\|_{0}^{2} .
\end{aligned}
$$

Now, we estimate the boundedness of $\mathcal{A}_{3}$. We have

$$
\begin{aligned}
\left\|\eta^{n+1}-\eta^{n}\right\|_{0} & =\left(\int_{\Omega}\left(\eta^{n+1}-\eta^{n}\right)^{2} d x\right)^{\frac{1}{2}}=\left(\int_{\Omega}\left|\int_{t_{n}}^{t_{n+1}} \frac{\partial \eta}{\partial t}(x, \theta) d \theta\right|^{2} d x\right)^{\frac{1}{2}} \\
& \leq \sqrt{\tau}\left(\int_{\Omega} \int_{t_{n}}^{t_{n+1}}\left|\frac{\partial \eta}{\partial t}\right|^{2}(x, \theta) d \theta d x\right)^{\frac{1}{2}} \\
& \leq \sqrt{\tau}\left\|\frac{\partial \eta}{\partial t}\right\|_{L^{2}\left(\left[t_{n}, t_{n+1}\right]: L^{2}(\Omega)\right)} .
\end{aligned}
$$


By the definition of $\hat{\mathcal{X}}_{x}^{n+1}\left(t_{n}\right)$, we can get

$$
J\left(\hat{\mathcal{X}}_{x}^{n+1}\left(t_{n}\right)\right)=\left(\begin{array}{cc}
1-\partial_{x} u_{h 1}^{n-1} \tau & -\partial_{y} u_{h 1}^{n-1} \tau \\
-\partial_{x} u_{h 2}^{n-1} \tau & 1-\partial_{y} u_{h 2}^{n-1} \tau
\end{array}\right)
$$

Hence,

$$
\operatorname{det} J\left(\hat{\mathcal{X}}_{x}^{n+1}\left(t_{n}\right)\right)=1+\mathcal{O}(\tau)
$$

Then we get

$$
\begin{aligned}
\left\|\eta^{n}-\dot{\eta}^{n}\right\|_{-1}= & \sup _{v \in V}\left(\|\nabla v\|_{0}^{-1}\left(\eta^{n}-\hat{\eta}^{n}, v\right)\right) \\
= & \sup _{v \in V}\left[\|\nabla v\|_{0}^{-1}\left(\int_{\Omega} \eta^{n}(x) v(x) d x-\int_{\Omega} \eta^{n}(z) v\left(\hat{\mathcal{X}}_{x}^{n}\left(t_{n}\right)^{-1}\right)\left(1+\mathcal{O}\left(\Delta t^{2}\right)\right) d z\right)\right] \\
\leq & \sup _{v \in V}\left(\|\nabla v\|_{0}^{-1} \int_{\Omega} \eta^{n-1}(x)\left(v(x)-v\left(\hat{\mathcal{X}}_{x}^{n}\left(t_{n}\right)^{-1}\right)\right) d x\right) \\
& +\sup _{v \in V}\left(C \tau^{2}\|\nabla v\|_{0}^{-1} \int_{\Omega} \eta^{n-1}(z) v\left(\hat{\mathcal{X}}_{x}^{n}\left(t_{n}\right)^{-1}\right) d z\right) .
\end{aligned}
$$

Let $G(x)=x-\hat{\mathcal{X}}_{x}^{n+1}\left(t_{n}\right)^{-1}$, then $|G(x)| \leq C \tau$, and

$$
\begin{aligned}
\left\|v(x)-v\left(\hat{\mathcal{X}}_{x}^{n+1}\left(t_{n}\right)^{-1}\right)\right\|_{0}^{2} & \leq \int_{\Omega}\left(\int_{t_{n}}^{t_{n+1}} \frac{d}{d t} v\left(\hat{\mathcal{X}}_{x}^{n+1}(t)^{-1}\right) d t\right)^{2} d x \\
& \leq C \tau^{2}\|\nabla v\|_{0}^{2}
\end{aligned}
$$

Similarly, we have

$$
\left\|v\left(\hat{\mathcal{X}}_{x}^{n+1}\left(t_{n}\right)^{-1}\right)\right\| \leq\|v\|_{0}^{2}(1+C \tau) .
$$

Then we deduce

$$
\left\|\eta^{n}-\dot{\eta}^{n}\right\|_{-1} \leq C \tau\left\|\eta^{n}\right\|_{0} .
$$

By (30) and (31), we have

$$
\left\|\eta^{n+1}-\dot{\eta}^{n}\right\|_{-1} \leq C \tau h^{k+1}\left\|u_{h}^{n}\right\|_{\infty}+C h^{k+1} \sqrt{\tau} .
$$

Therefore, we get

$$
\begin{aligned}
\mathcal{A}_{3} & \leq\left\|\eta^{n+1}-\dot{\eta}^{n}\right\|_{-1}\left\|\nabla \hat{\xi}_{h}^{n+1}\right\|_{0} \\
& \leq C \tau^{2} h^{2(k+1)}+C \tau h^{2(k+1)}+\frac{\nu \tau}{16}\left\|\nabla \hat{\xi}_{h}^{n+1}\right\|_{0}^{2} .
\end{aligned}
$$

Similarly, we obtain

$$
\mathcal{A}_{4} \leq C \tau\left\|\xi_{h}^{n}\right\|_{0}^{2}+\frac{\nu \tau}{16}\left\|\nabla \hat{\xi}_{h}^{n+1}\right\|_{0}^{2}
$$


For the term $\mathcal{A}_{5}$, by Taylor's formula, we can get $\left\|T^{n+1}-T^{n}\right\|_{0} \leq C \tau$, then

$$
\begin{aligned}
\mathcal{A}_{5} & =k v^{2} g \tau\left(T^{n+1}-T_{h}^{n}, \hat{\xi}_{h}^{n+1}\right) \\
& =k v^{2} g \tau\left(T^{n+1}-T^{n}, \hat{\xi}_{h}^{n+1}\right)+k v^{2} g \tau\left(T^{n}-T_{h}^{n}, \hat{\xi}_{h}^{n+1}\right) \\
& \leq C k^{2} v^{4} \tau^{3}+C k^{2} v^{4} \tau\left\|T^{n}-T_{h}^{n}\right\|_{0}^{2}+\frac{\nu \tau}{8}\left\|\nabla \xi_{h}^{n+1}\right\|_{0}^{2} .
\end{aligned}
$$

Combining (27), (28), (20), and (34), we arrive at

$$
\begin{aligned}
& \left\|\hat{\xi}_{h}^{n+1}\right\|_{0}^{2}-\left\|\xi_{h}^{n}\right\|_{0}^{2}+\left\|\hat{\xi}_{h}^{n+1}-\xi_{h}^{n}\right\|_{0}^{2}+\frac{1}{2} \nu \tau\left\|\nabla \hat{\xi}_{h}^{n+1}\right\|_{0}^{2}+\nu \tau\left(\left\|s_{h}^{n+1}\right\|_{0}^{2}-\left\|s_{h}^{n}\right\|_{0}^{2}\right) \\
& \leq C \tau\left(\tau^{2}+h^{2(k+1)}\right)+C \tau\left\|\xi_{h}^{n}\right\|_{0}^{2}+C k^{2} \nu^{4} \tau\left\|T^{n}-T_{h}^{n}\right\|_{0}^{2} .
\end{aligned}
$$

Subtracting $\tau^{-1}\left(r_{h} T^{n+1}-r_{h} \dot{T}^{n}, \psi_{h}\right)+\lambda v\left(\nabla r_{h} T^{n+1}, \nabla \psi_{h}\right)$ from both sides of (7), we can get

$$
\begin{aligned}
( & \left.\frac{\left(T_{h}^{n+1}-r_{h} T^{n+1}\right)-\left(\dot{T}_{h}^{n}-r_{h} \dot{T}^{n}\right)}{\tau}, \psi_{h}\right)+\lambda \nu\left(\nabla\left(T_{h}^{n+1}-r_{h} T^{n+1}\right), \nabla \psi_{h}\right) \\
= & -\left(\frac{r_{h} T^{n+1}-r_{h} \dot{T}^{n}}{\tau}, \psi_{h}\right)-\lambda \nu\left(\nabla r_{h} T^{n+1}, \nabla \psi_{h}\right)+\left(b\left(t_{n+1}\right), \nabla \psi_{h}\right) \\
= & \left(\frac{\left(T^{n+1}-r_{h} T^{n+1}\right)-\left(\dot{T}^{n}-r_{h} \dot{T}^{n}\right)}{\tau}, \psi_{h}\right)+\lambda \nu\left(\nabla\left(T^{n+1}-r_{h} T^{n+1}\right), \nabla \psi_{h}\right) \\
& \quad+\left(\zeta\left(t_{n+1}\right), \psi_{h}\right) .
\end{aligned}
$$

Letting $\dot{\varepsilon}_{h}^{n+1}=\dot{T}_{h}^{n+1}-r_{h} \dot{T}^{n+1}, \theta^{n+1}=T^{n+1}-r_{h} T^{n+1}, \dot{\theta}^{n}=\dot{T}^{n}-r_{h} \dot{T}^{n}$, and $\psi_{h}=2 \tau \varepsilon_{h}^{n+1}$ in (36), we can get

$$
\begin{aligned}
& 2\left(\varepsilon_{h}^{n+1}-\varepsilon_{h}^{n}, \varepsilon_{h}^{n+1}\right)+2 \lambda \nu \tau\left(\nabla \varepsilon_{h}^{n+1}, \nabla \varepsilon_{h}^{n+1}\right) \\
& \quad=2\left(\theta^{n+1}-\dot{\theta}^{n}, \varepsilon_{h}^{n+1}\right)+2\left(\zeta\left(t_{n+1}\right), \varepsilon_{h}^{n+1}\right)+2\left(\varepsilon_{h}^{n}-\dot{\varepsilon}_{h}^{n}, \varepsilon_{h}^{n+1}\right) .
\end{aligned}
$$

Similarly to (32), we get

$$
\begin{aligned}
& \left\|\varepsilon_{h}^{n}-\dot{\varepsilon}_{h}^{n}\right\|_{0} \leq C \tau\left\|\varepsilon_{h}^{n}\right\|_{0}, \\
& \left\|\theta^{n+1}-\dot{\theta}^{n}\right\|_{-1} \leq C \tau h^{k+1}\left\|u_{h}^{n}\right\|_{\infty}+C h^{k+1} \sqrt{\tau} .
\end{aligned}
$$

Then we deduce

$$
\begin{gathered}
\left\|\varepsilon_{h}^{n+1}\right\|_{0}^{2}-\left\|\varepsilon_{h}^{n}\right\|_{0}^{2}+\left\|\varepsilon_{h}^{n+1}-\varepsilon_{h}^{n}\right\|_{0}+2 \lambda \nu \tau\left\|\nabla \varepsilon_{h}^{n+1}\right\|_{0}^{2} \\
\leq C \tau h^{r+1}+C \tau^{3}+\lambda \nu \tau\left\|\nabla \varepsilon_{h}^{n+1}\right\|_{0}^{2}+C \tau\left\|\varepsilon_{h}^{n}\right\|_{0}^{2} .
\end{gathered}
$$

Namely,

$$
\begin{aligned}
& \left\|\varepsilon_{h}^{n+1}\right\|_{0}^{2}-\left\|\varepsilon_{h}^{n}\right\|_{0}^{2}+\left\|\varepsilon_{h}^{n+1}-\varepsilon_{h}^{n}\right\|_{0}+\lambda \nu \tau\left\|\nabla \varepsilon_{h}^{n+1}\right\|_{0}^{2} \\
& \quad \leq C \tau h^{2(r+1)}+C \tau^{3}+C \tau\left\|\varepsilon_{h}^{n}\right\|_{0}^{2} .
\end{aligned}
$$


Adding (35) to (37), we get

$$
\begin{gathered}
\left\|\xi_{h}^{n+1}\right\|_{0}^{2}-\left\|\xi_{h}^{n}\right\|_{0}^{2}+\left\|\xi_{h}^{n+1}-\xi_{h}^{n}\right\|_{0}^{2}+\frac{1}{2} \nu \tau\left\|\nabla \hat{\xi}_{h}^{n+1}\right\|_{0}^{2} \\
+\left\|\varepsilon_{h}^{n+1}\right\|_{0}^{2}-\left\|\varepsilon_{h}^{n}\right\|_{0}^{2}+\left\|\varepsilon_{h}^{n+1}-\varepsilon_{h}^{n}\right\|_{0}+\lambda \nu \tau\left\|\nabla \varepsilon_{h}^{n+1}\right\|_{0}^{2} \\
\leq C \tau h^{2(r+1)}+C \tau^{3}+C \tau\left\|\xi_{h}^{n}\right\|_{0}^{2}+C \tau\left\|\varepsilon_{h}^{n}\right\|_{0}^{2} .
\end{gathered}
$$

Summing over $n$ from 0 to $N$ gives

$$
\begin{aligned}
\left\|\hat{\xi}_{h}^{N+1}\right\|_{0}^{2}+\sum_{n=0}^{N}\left\|\hat{\xi}_{h}^{n+1}-\xi_{h}^{n}\right\|_{0}^{2}+2 \sum_{n=0}^{N}\left\|\nabla \rho_{h}^{n+1}\right\|_{0}^{2} \\
\quad+\frac{1}{2} \nu \tau \sum_{n=0}^{N}\left\|\nabla \hat{\xi}_{h}^{n+1}\right\|_{0}^{2}+\left\|\varepsilon_{h}^{N+1}\right\|_{0}^{2} \\
+\sum_{n=0}^{N}\left\|\varepsilon_{h}^{n+1}-\varepsilon_{h}^{n}\right\|_{0}^{2}+\lambda \nu \tau \sum_{n=0}^{N}\left\|\nabla \varepsilon_{h}^{n+1}\right\|_{0}^{2} \\
\leq C\left(\tau^{2}+h^{2(k+1)}\right)+C \tau \sum_{n=0}^{N}\left(\left\|\varepsilon_{h}^{n}\right\|_{0}^{2}+\left\|\xi_{h}^{n}\right\|_{0}^{2}\right) .
\end{aligned}
$$

By Gronwall lemma, we obtain

$$
\begin{gathered}
\left\|\hat{\xi}_{h}^{N+1}\right\|_{0}^{2}+\sum_{n=0}^{N}\left\|\hat{\xi}_{h}^{n+1}-\xi_{h}^{n}\right\|_{0}^{2}+\frac{1}{2} \nu \tau \sum_{n=0}^{N}\left\|\nabla \hat{\xi}_{h}^{n+1}\right\|_{0}^{2}+\left\|\varepsilon_{h}^{N+1}\right\|_{0}^{2} \\
+\sum_{n=0}^{N}\left\|\varepsilon_{h}^{n+1}-\xi_{h}^{n}\right\|_{0}^{2}+\lambda \nu \tau \sum_{n=0}^{N}\left\|\nabla \varepsilon_{h}^{n+1}\right\|_{0}^{2} \leq C\left(\tau^{2}+h^{2(k+1)}\right) .
\end{gathered}
$$

Using (6), we get

$$
\left(\frac{\xi_{h}^{n+1}-\hat{\xi}_{h}^{n+1}}{\tau}, v_{h}\right)-b\left(p_{h}^{n+1}, v_{h}\right)=0
$$

Let $v_{h}=2 \tau \xi_{h}^{n+1}$, we can get

$$
\left\|\xi_{h}^{n+1}\right\|_{0}^{2}-\left\|\hat{\xi}_{h}^{n+1}\right\|_{0}^{2}+\left\|\xi_{h}^{n+1}-\hat{\xi}_{h}^{n+1}\right\|_{0}^{2}=0
$$

Then we have

$$
\begin{array}{r}
\left\|\xi_{h}^{N+1}\right\|_{0}^{2}+\sum_{n=0}^{N}\left\|\hat{\xi}_{h}^{n+1}-\xi_{h}^{n}\right\|_{0}^{2}+\frac{1}{2} \nu \tau \sum_{n=0}^{N}\left\|\nabla \hat{\xi}_{h}^{n+1}\right\|_{0}^{2}+\left\|\varepsilon_{h}^{N+1}\right\|_{0}^{2} \\
+\sum_{n=0}^{N}\left\|\varepsilon_{h}^{n+1}-\xi_{h}^{n}\right\|_{0}^{2}+\lambda \nu \tau \sum_{n=0}^{N}\left\|\nabla \varepsilon_{h}^{n+1}\right\|_{0}^{2} \leq C\left(\tau^{2}+h^{2(k+1)}\right) .
\end{array}
$$

Using the inequality (see [41], Remark 1.6 and [19]),

$$
\left\|\nabla P_{h} u\right\|_{0} \leq C\|\nabla u\|_{0}
$$


we have

$$
\begin{gathered}
\left\|\xi_{h}^{N+1}\right\|_{0}^{2}+\sum_{n=0}^{N}\left\|\hat{\xi}_{h}^{n+1}-\xi_{h}^{n}\right\|_{0}^{2}+\frac{1}{2} \nu \tau \sum_{n=0}^{N}\left\|\nabla \xi_{h}^{n+1}\right\|_{0}^{2}+\left\|\varepsilon_{h}^{N+1}\right\|_{0}^{2} \\
+\sum_{n=0}^{N}\left\|\varepsilon_{h}^{n+1}-\xi_{h}^{n}\right\|_{0}^{2}+\lambda \nu \tau \sum_{n=0}^{N}\left\|\nabla \varepsilon_{h}^{n+1}\right\|_{0}^{2} \leq C\left(\tau^{2}+h^{2(k+1)}\right) .
\end{gathered}
$$

Using the triangle inequality, we deduce

$$
\left\|u_{h}^{n+1}\right\|_{W^{1, \infty}} \leq\left\|u_{h}^{n+1}-R_{h}^{n+1}\right\|_{W^{1, \infty}}+\left\|R_{h}^{n+1}\right\|_{W^{1, \infty}} .
$$

Via the inverse inequality, $\left\|v_{h}\right\|_{W^{1, \infty}} \leq C h^{-1}\left\|\nabla v_{h}\right\|_{0}$ (see [36]), we can get

$$
\left\|u_{h}^{n+1}\right\|_{W^{1, \infty}} \leq C h^{-1}\left\|\nabla\left(u_{h}^{n+1}-R_{h}^{n+1}\right)\right\|_{0}+\left\|R_{h}^{n+1}\right\|_{\infty} .
$$

We thus finish the proof.

Theorem 4.6 (Error estimates for the velocity and temperature) If $\tau \leq \frac{1}{2 L_{n}}, u, p, u_{t}$, and $p_{t}$ are sufficiently smooth, we have

$$
\begin{aligned}
& \tau \sum_{n=0}^{N}\left\|u^{N+1}-u_{h}^{N+1}\right\|_{0}^{2} \leq C\left(\tau^{2}+h^{2(k+1)}\right), \\
& \frac{1}{2} \nu \tau \sum_{n=0}^{N}\left\|\nabla\left(u^{n+1}-u_{h}^{n+1}\right)\right\|_{0}^{2} \leq C\left(\tau^{2}+h^{2 k}\right), \\
& \tau \sum_{n=0}^{N}\left\|T^{N+1}-T_{h}^{N+1}\right\|_{0}^{2} \leq C\left(\tau^{2}+h^{2(k+1)}\right), \\
& \frac{1}{2} \nu \tau \sum_{n=0}^{N}\left\|\nabla\left(T^{n+1}-T_{h}^{n+1}\right)\right\|_{0}^{2} \leq C\left(\tau^{2}+h^{2 k}\right) .
\end{aligned}
$$

Proof Using triangle inequality, (23), and Lemma 4.3, we can get this theorem.

\subsection{Error estimates for the pressure}

The following theorem on the pressure is a consequence of the previous theorem on the velocity.

Theorem 4.7 (Error estimate for pressure) If $\tau \leq \frac{1}{2 L_{n}}, u, p, u_{t}$, and $p_{t}$ are sufficiently smooth, we have for all $1 \leq n \leq N$,

$$
\left\|p^{n+1}-p_{h}^{n+1}\right\|_{0} \leq C\left(\tau+h^{k}\right)
$$

Proof By (6), we deduce

$$
\begin{aligned}
& \left(p^{n+1}-p_{h}^{n+1}, \nabla v_{h}\right) \\
& \quad=\left(\frac{u^{n+1}-\bar{u}^{n}}{\tau}-v \Delta u^{n+1}+\nabla p^{n+1}-k v^{2} g T^{n+1}-f^{n+1}, v_{h}\right)-\left(\frac{\xi_{h}^{n+1}-\xi_{h}^{n}}{\tau}, v_{h}\right)
\end{aligned}
$$




$$
\begin{aligned}
& -v\left(\nabla \hat{\xi}_{h}^{n+1}, \nabla v_{h}\right)+\left(\frac{\eta^{n+1}-\dot{\eta}^{n}}{\tau}, v_{h}\right) \\
& +\left(\frac{\dot{u}^{n}-\bar{u}^{n}}{\tau}, v_{h}\right)+\left(\frac{\dot{\xi}_{h}^{n}-\xi_{h}^{n}}{\tau}, v_{h}\right) \\
& +v\left(\nabla\left(u^{n+1}-R_{h}^{n+1}\right), \nabla v_{h}\right)+k v^{2} g\left(T^{n+1}-T_{h}^{n}, v_{h}\right) .
\end{aligned}
$$

By the LBB condition and Cauchy-Schwarz inequality, we get

$$
\begin{aligned}
& \left\|p^{n+1}-p_{h}^{n+1}\right\|_{0} \\
& \leq\left\|\frac{u^{n+1}-\bar{u}^{n}}{\tau}-v \Delta u^{n+1}+\nabla p^{n+1}-k v^{2} g T^{n+1}-f^{n+1}\right\|_{0} \\
& \quad+C \tau^{-1}\left\|\xi_{h}^{n+1}-\xi_{h}^{n}\right\|_{0}+v\left\|\hat{\xi}_{h}^{n+1}\right\|_{0}+C\left\|\frac{\dot{u}^{n}-\bar{u}^{n}}{\tau}\right\|_{0} \\
& \quad+C\left\|\frac{\eta^{n+1}-\dot{\eta}^{n}}{\tau}\right\|_{0}+C\left\|\frac{\dot{\xi}_{h}^{n}-\xi_{h}^{n}}{\tau}\right\|_{0}-v\left\|\nabla\left(u^{n+1}-R_{h}^{n+1}\right)\right\|_{0} \\
& \quad+k v^{2} g\left\|T^{n+1}-T_{h}^{n}\right\|_{0} .
\end{aligned}
$$

Using (27), (28), (20), and (34), we arrive at

$$
\left\|p^{n+1}-p_{h}^{n+1}\right\|_{0} \leq C\left(\tau+h^{k}\right)
$$

Thus, we finish the proof.

\section{Numerical experiments}

In order to show the effect of our method, we give some numerical results in this section.

\subsection{Bénard convection problem}

The first experiments is Bénard convection problem in the domain $\Omega=[0,5] \times[0,1]$ with the forcing $f=0$ and $b=0$. Figure 1 displays the initial and boundary conditions for velocity $u$ and temperature $T$. It means that the boundary conditions for the velocity are the no-slip boundary condition $u=0$ on $\partial \Omega$, thermal insulation $\partial_{v} T=0$ on the lateral boundaries, and a fixed temperature difference between top and bottom boundaries. Here, we choose $h=1 / 16, \tau=0.01$, and the finite element space is a Taylor-Hood finite element space. Here, we use the software package FreeFEM++ [42] for our program.

First, we set $\kappa=10^{4}, \lambda=1.0, v=1$.0. Figure 2 gives the numerical temperature at $t=$ $0.05,0.1,0.15$, and 1.0. Figure 3 gives the numerical pressure at $t=0.05,0.1,0.15$, and 1.0. Figure 4 gives the numerical streamline at $t=0.05,0.1,0.15$, and 1.0 .

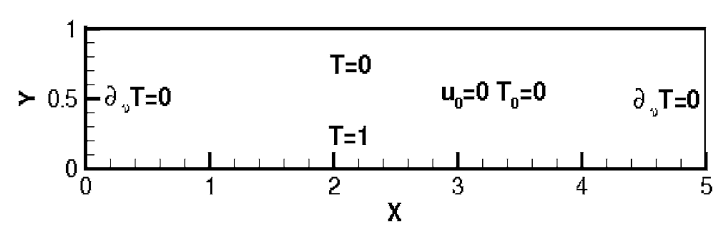

Figure 1 Physics model of Bénard convection problem. 


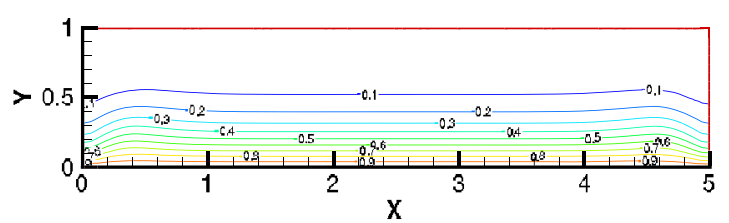

(a) $t=0.05$

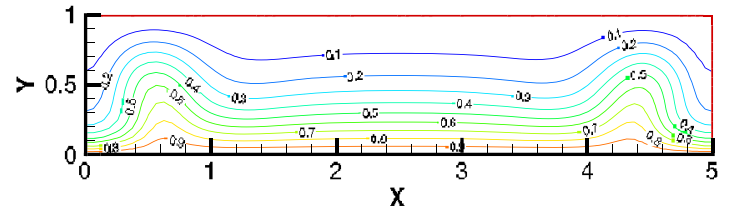

(b) $\mathrm{t}=0.1$

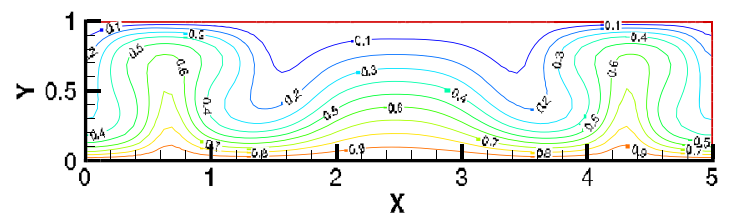

(c) $t=0.15$

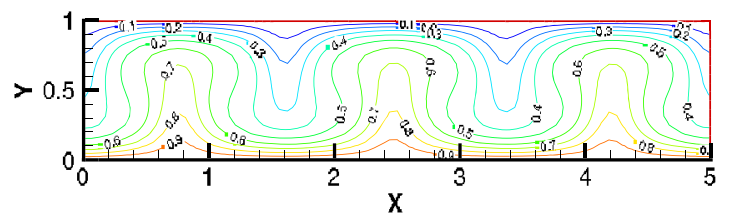

(d) $t=1$

Figure 2 Numerical temperatures of Bénard convection problem with $\kappa=1 \times 10^{4}$ at different times.

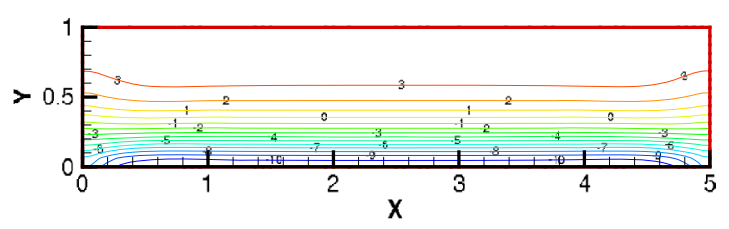

(a) $t=0.05$

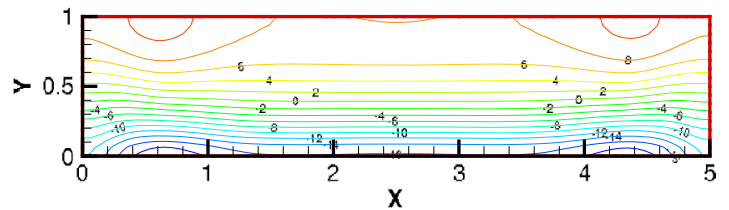

(b) $t=0.1$

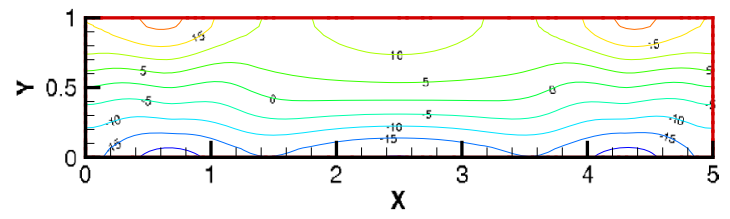

(c) $\mathrm{t}=0.15$

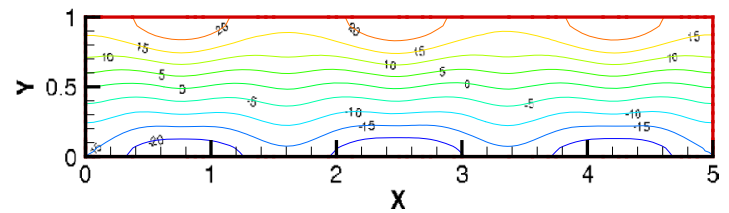

(d) $\mathrm{t}=1$

Figure 3 Numerical pressures of Bénard convection problem with $\kappa=1 \times 10^{4}$ at different times. 


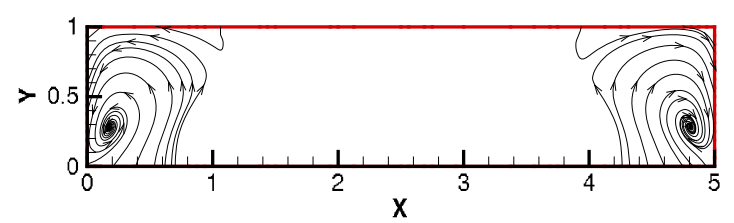

(a) $\mathrm{t}=0.05$

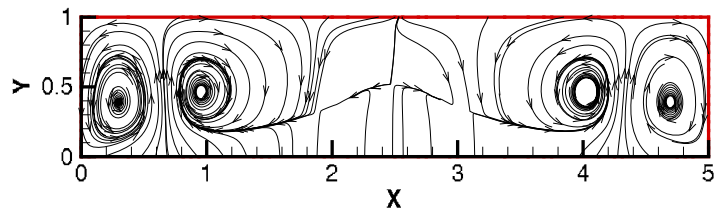

(b) $\mathrm{t}=0.1$

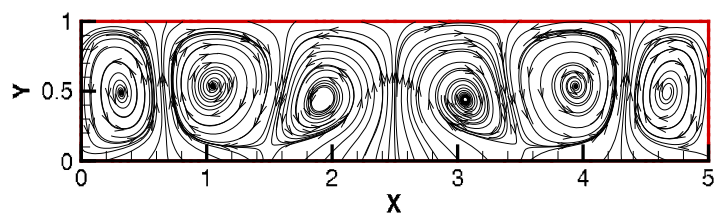

(c) $\mathrm{t}=0.15$

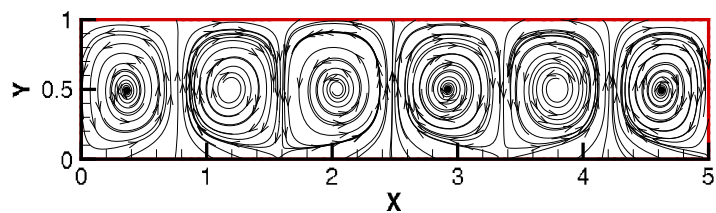

(d) $t=1$

Figure 4 Numerical streamlines of Bénard convection problem with $\kappa=1 \times 10^{4}$ at different times.

Then we set $\kappa=10^{5}, \lambda=1.0, v=1.0$. Figure 5 gives the numerical temperature at $t=0.05,0.1,0.15$, and 1.0. Figure 6 gives the numerical pressure at $t=0.05,0.1,0.15$, and 1 . Figure 7 gives the numerical streamline at $t=0.05,0.1,0.15$, and 1 . From the numerical results, we can see that MCPFEM can simulate the fluid field, temperature field and pressure field very well, and it works well for a high Grashoff number $\kappa$.

\subsection{Thermal driven cavity flow problem}

Here, we consider the thermal driven flow in an enclosed square $\Omega=[0,1]^{2}$ with the forc$\operatorname{ing} f=0$ and $b=0$, and the initial and boundary conditions are given by Figure 8. It means that the boundary conditions for velocity is no-slip boundary condition $u=0$ on $\partial \Omega$, and thermal insulation $\partial_{v} T=0$ on the top and bottom boundaries, and a fixed temperature difference between left and right boundaries. Here, we choose $h=1 / 32, \tau=10^{-4}$, and the finite element space is a Taylor-Hood finite element space.

We choose $\lambda=1, v=1, \kappa=10^{5}$ and $10^{6}$ respectively. Figures 9 and 10 give the numerical results for $\kappa=10^{5}$ and $10^{6}$, respectively. From the numerical results, we can see that MCPFEM can simulate the fluid field, temperature field, and pressure field very well. The numerical experiments confirm our theoretical analysis and demonstrate the efficiency of our method. 


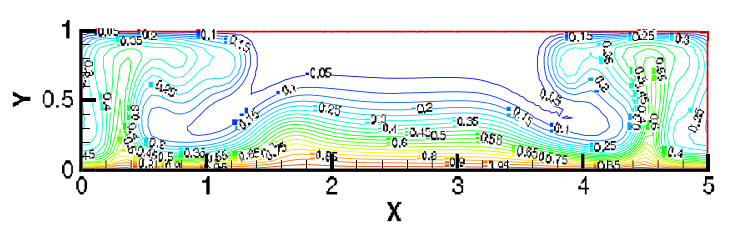

(a) $t=0.05$

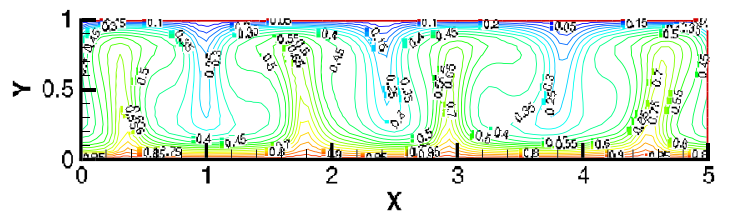

(b) $t=0.1$

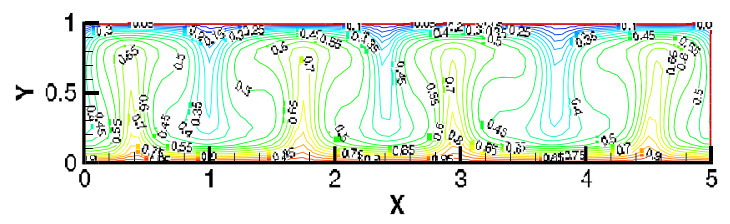

(c) $t=0.15$

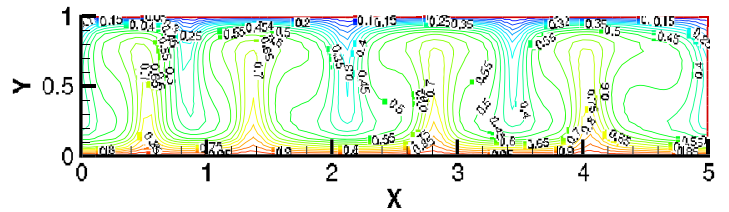

(d) $\mathrm{t}=1$

Figure 5 Numerical temperatures of Bénard convection problem with $\kappa=1 \times 10^{5}$ at different times.

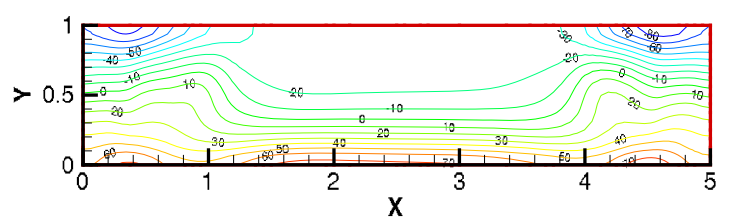

(a) $t=0.05$

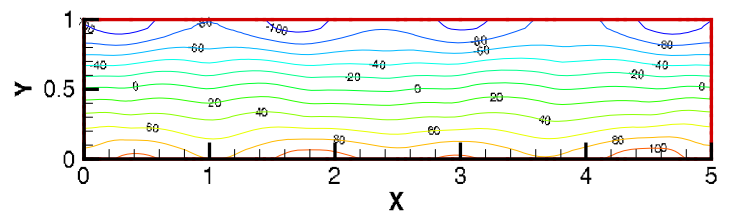

(b) $t=0.1$

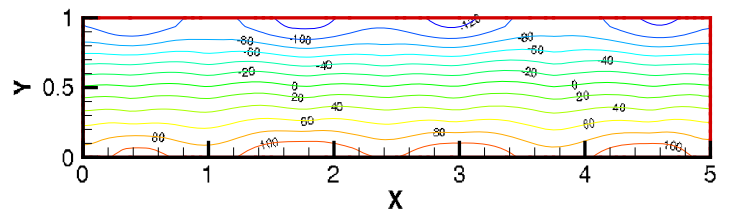

(c) $\mathrm{t}=0.15$

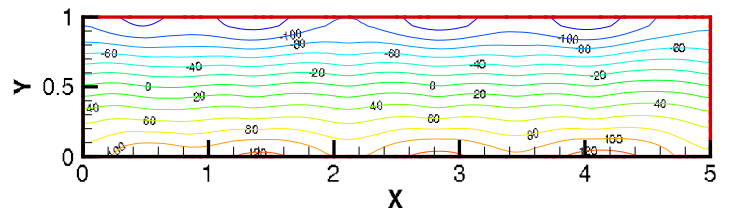

(d) $\mathrm{t}=1$

Figure 6 Numerical pressures of Bénard convection problem with $\kappa=1 \times 10^{5}$ at different times. 


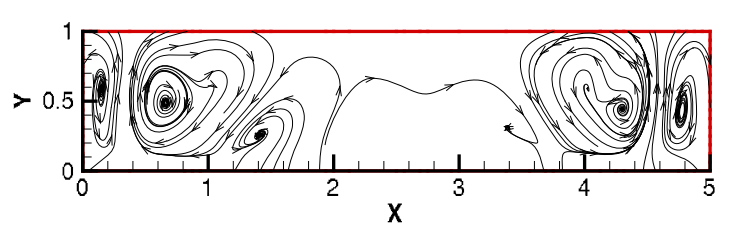

(a) $t=0.05$

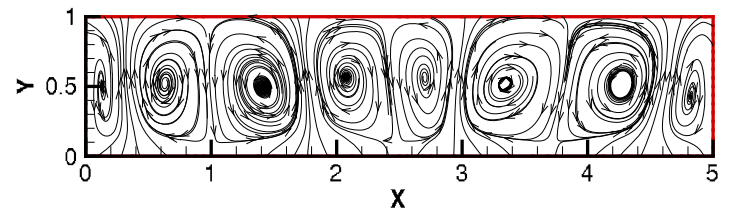

(b) $\mathrm{t}=0.1$

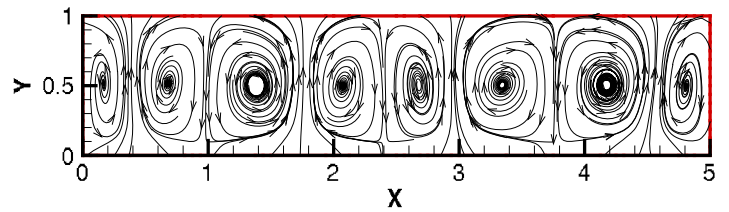

(c) $\mathrm{t}=0.15$

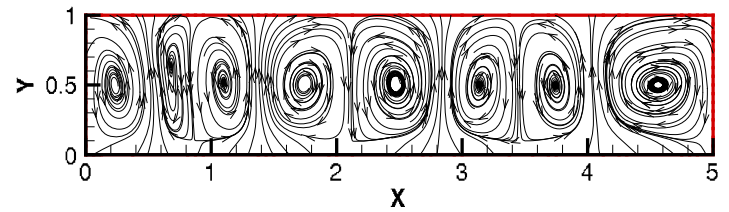

(d) $\mathrm{t}=1$

Figure 7 Numerical streamline of Bénard convection problem with $\kappa=1 \times 10^{5}$ at different times.

Figure 8 Physics model of thermal driven cavity flow.

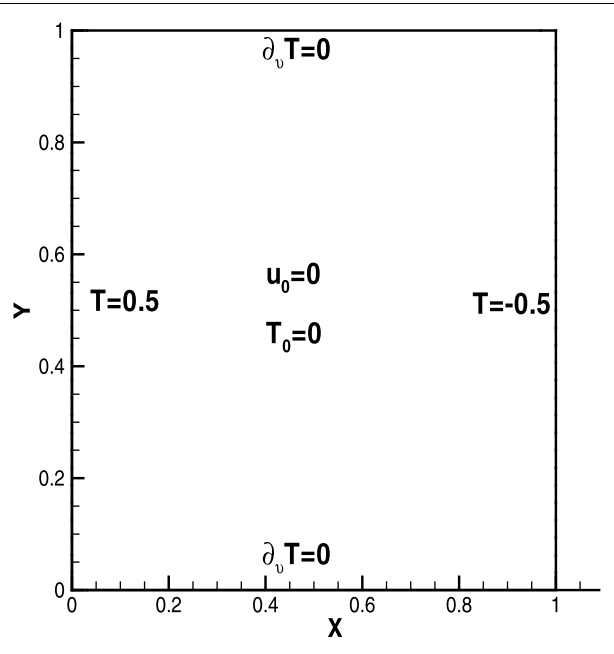




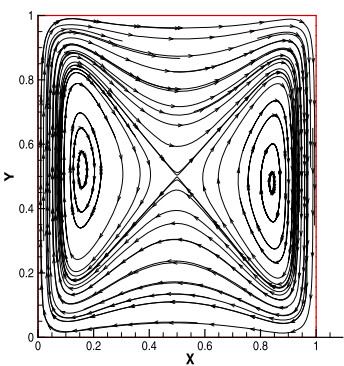

(a) $\mathrm{t}=0.003$

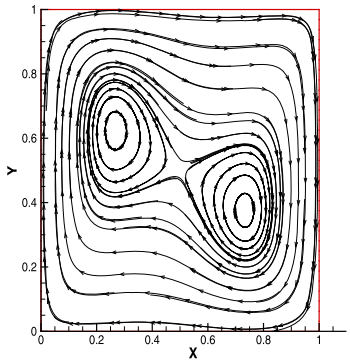

(d) $t=0.01$

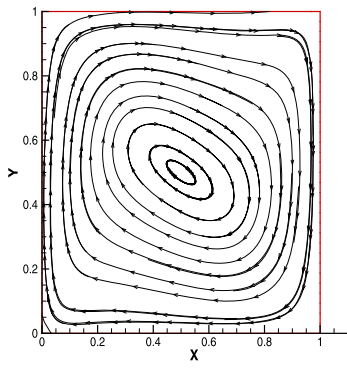

(g) $\mathrm{t}=0.025$

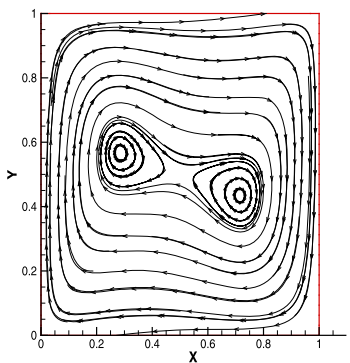

(j) $\mathrm{t}=0.1$

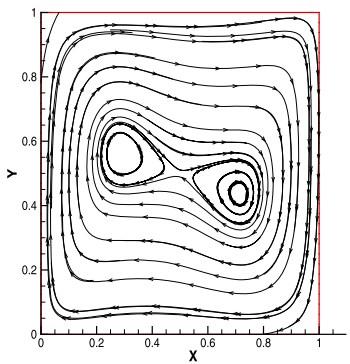

(m) $\mathrm{t}=0.2$

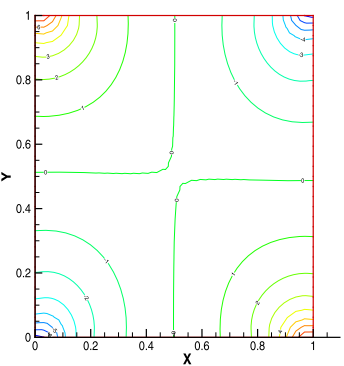

(b) $\mathrm{t}=0.003$

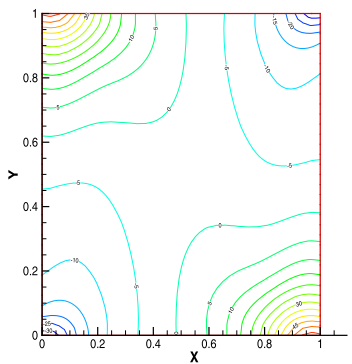

(e) $\mathrm{t}=0.01$

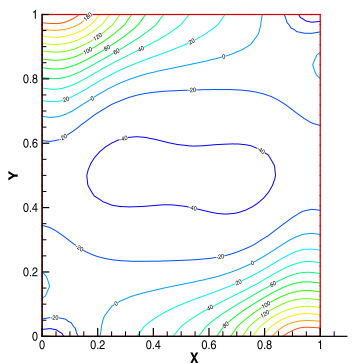

(h) $\mathrm{t}=0.025$

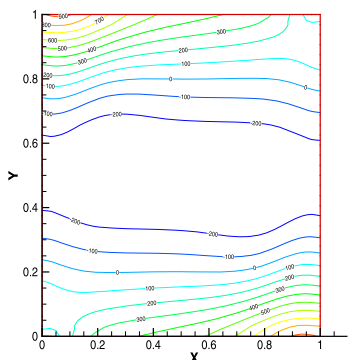

(k) $\mathrm{t}=0.1$

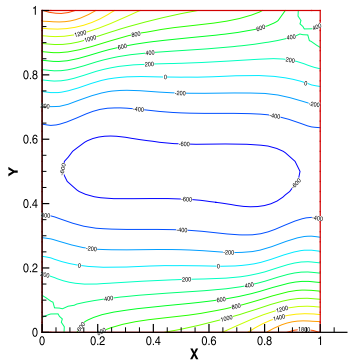

(n) $\mathrm{t}=0.2$

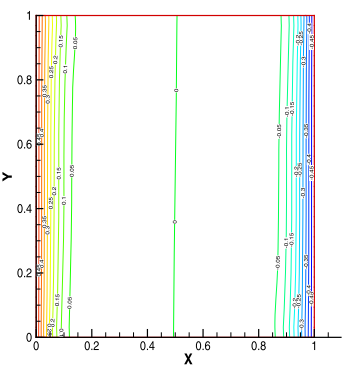

(c) $\mathrm{t}=0.003$

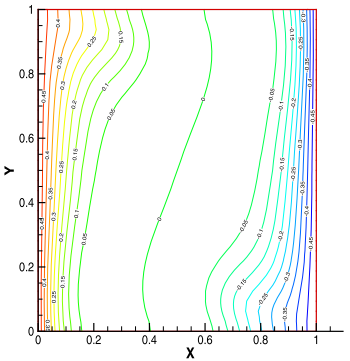

(f) $\mathrm{t}=0.01$

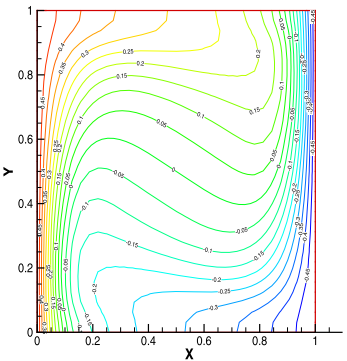

(i) $\mathrm{t}=0.025$

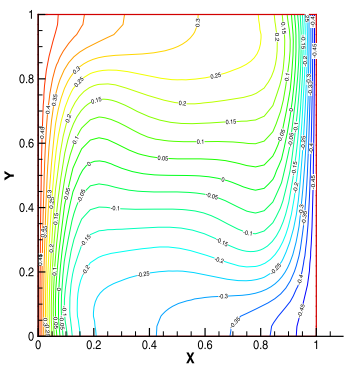

(l) $\mathrm{t}=0.1$

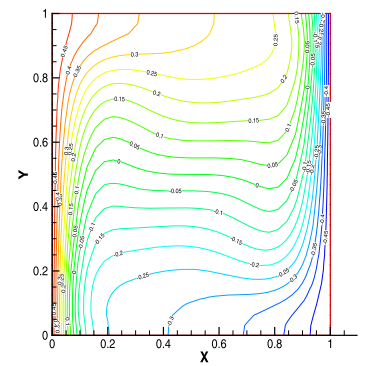

(o) $\mathrm{t}=0.2$

Figure 9 Numerical results of thermal driven cavity flow with $\kappa=1 \times 10^{5}$ at different time, left panels the numerical streamlines, middle panels the numerical pressures, and right panels the numerical temperatures. 


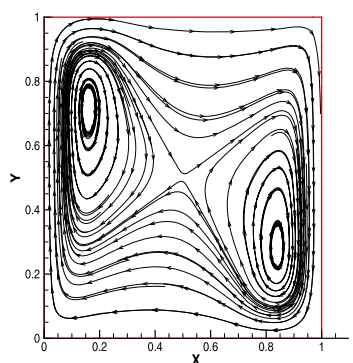

(a) $\mathrm{t}=0.003$

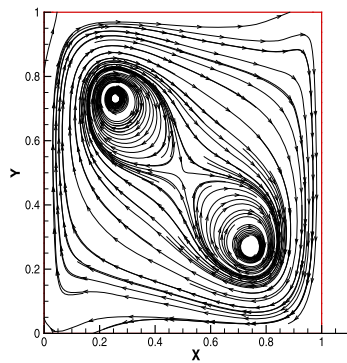

(d) $\mathrm{t}=0.01$

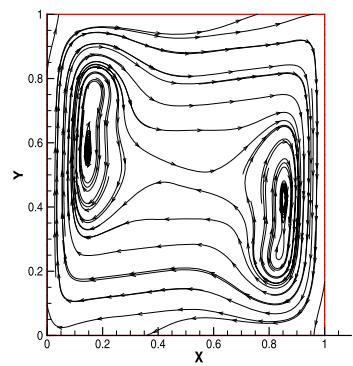

(g) $\mathrm{t}=0.025$

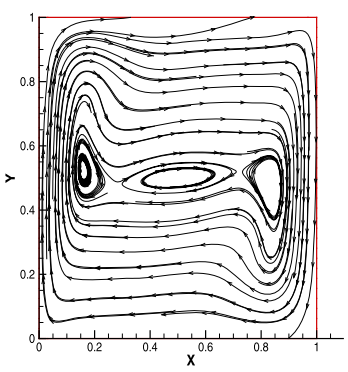

(j) $\mathrm{t}=0.1$

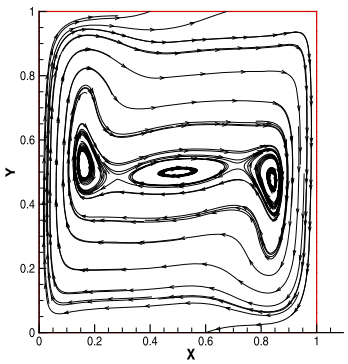

(m) $\mathrm{t}=0.2$

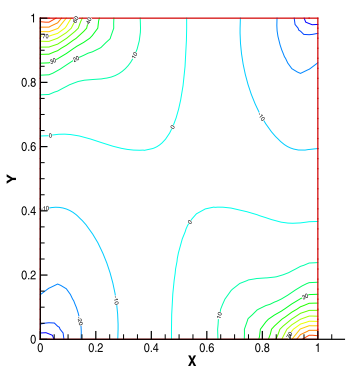

(b) $t=0.003$

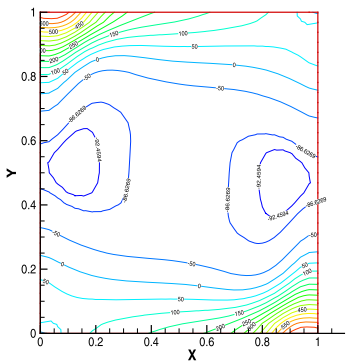

(e) $t=0.01$

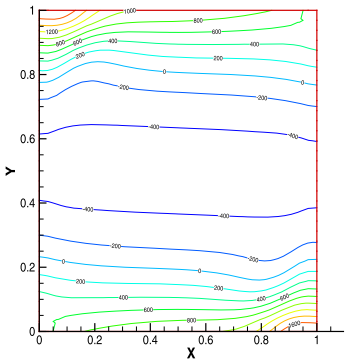

(h) $\mathrm{t}=0.025$

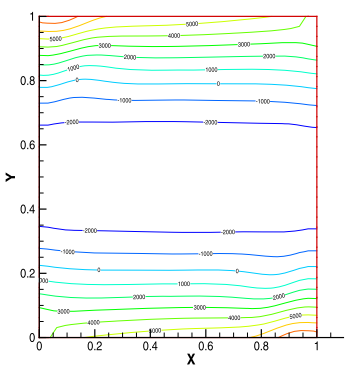

(k) $\mathrm{t}=0.1$

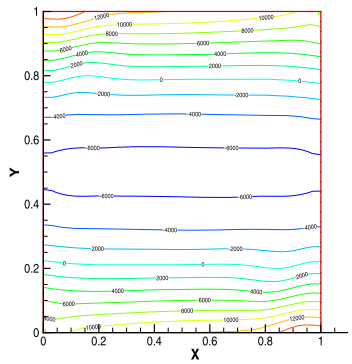

(n) $\mathrm{t}=0.2$

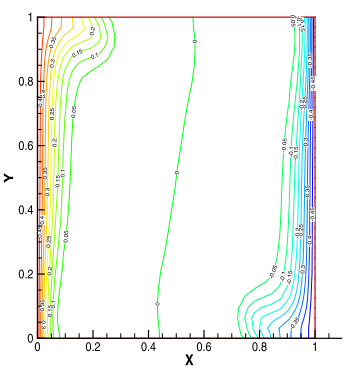

(c) $\mathrm{t}=0.003$

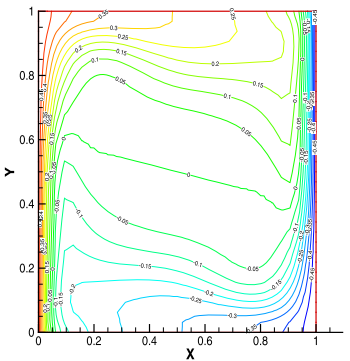

(f) $\mathrm{t}=0.01$

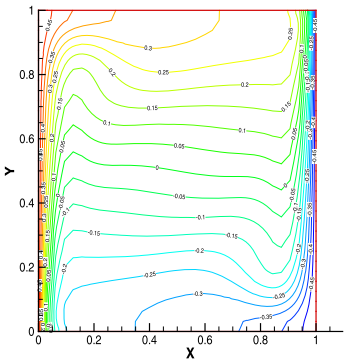

(i) $\mathrm{t}=0.025$

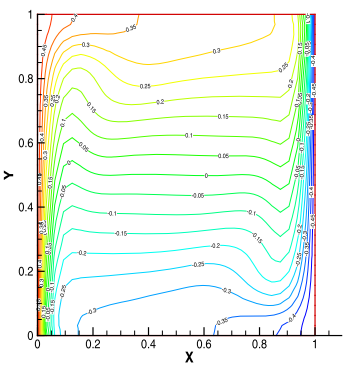

(l) $\mathrm{t}=0.1$

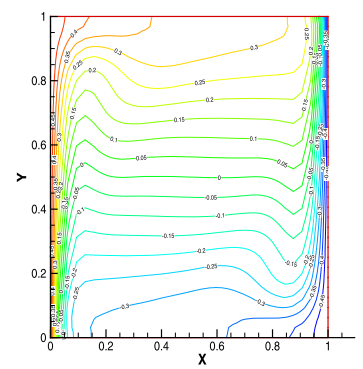

(o) $\mathrm{t}=0.2$

Figure 10 Numerical results of thermal driven cavity flow with $\kappa=1 \times 10^{6}$ at different times, left panels the numerical streamlines, middle panels the numerical pressures, and right panels the numerical temperatures. 


\section{Competing interests}

The authors declare that they have no competing interests.

\section{Authors' contributions}

The authors contributed equally in this article. They read and approved the final manuscript.

\section{Author details}

${ }^{1}$ School of Mathematics and Information Science, Henan Polytechnic University, Jiaozuo, 454003, P.R. China. ${ }^{2}$ School of Materials Science and Engineering, Henan Polytechnic University, Jiaozuo, 454003, P.R. China.

\section{Acknowledgements}

The authors would like to thank the anonymous referees for their valuable suggestions and comments, which helped to improve the quality of the paper. This work of the authors was supported in part by Chinese NSF (Grant Nos. 11301156 and 11401177).

Received: 4 March 2015 Accepted: 19 August 2015 Published online: 01 September 2015

\section{References}

1. Luo, Z, Chen, J, Navon, IM, Zhu, J: An optimizing reduced PLSMFE formulation for non-stationary conduction-convection problems. Int. J. Numer. Methods Fluids 60, 409-436 (2009)

2. Garcia, J, Cabeza, J, Rodriguez, A: Two-dimensional non-linear inverse heat conduction problem based on the singular value decomposition. Int. J. Therm. Sci. 48, 1081-1093 (2009)

3. Kim, D, Choi, Y: Analysis of conduction-natural convection conjugate heat transfer in the gap between concentric cylinders under solar irradiation. Int. J. Therm. Sci. 48, 1247-1258 (2009)

4. Boland, J, Layton, W: Error analysis for finite element method for natural convection problems. Numer. Funct. Anal. Optim. 11, 449-483 (1990)

5. Si, ZY, He, YN: A coupled Newton iterative mixed finite element method for stationary conduction-convection problems. Computing 89, 1-25 (2010)

6. Si, ZY, Shang, YQ, Zhang, T: New one- and two-level Newton iterative mixed finite element methods for stationary conduction-convection problem. Finite Elem. Anal. Des. 47, 175-183 (2011)

7. $\mathrm{Si}, \mathrm{ZY}, \mathrm{Zhang}, \mathrm{T}$, Wang, $\mathrm{K}: \mathrm{A}$ Newton iterative scheme mixed finite element method for stationary conduction-convection problems. Int. J. Comput. Fluid Dyn. 24, 135-141 (2010)

8. $\mathrm{Si}, \mathrm{ZY}, \mathrm{He}, \mathrm{YN}$ : A defect-correction mixed finite element method for stationary conduction-convection problems. Math. Probl. Eng. 2011, Article ID 370192 (2011). doi:10.1155/2011/370192

9. Si, ZY, He, YN, Wang, K: A defect-correction method for unsteady conduction convection problems I: spatial discretization. Sci. China Math. 54, 185-204 (2011)

10. Si, ZY, He, YN, Zhang, T: A defect-correction method for unsteady conduction-convection problems II: time discretization. J. Comput. Appl. Math. 236, 2553-2573 (2012)

11. Chorin, AJ: Numerical solution of the Navier-Stokes equations. Math. Comput. 22, 745-762 (1968)

12. Temam, R: Sur l'approximation de la solution des équations de Navier-Stokes par la méthode des pas fractionnaires II. Arch. Ration. Mech. Anal. 33, 377-385 (1969)

13. Guermond, JL, Shen, J: On the error estimates of rotational pressure-correction projection methods. Math. Comput. 73, 1719-1737 (2004)

14. Sun, $\mathrm{HY}, \mathrm{He}, \mathrm{YN}$, Feng, $\mathrm{XL}$ : On error estimates of the pressure-correction projection methods for the time-dependent Navier-Stokes equations. Int. J. Numer. Anal. Model. 8, 70-85 (2011)

15. Guermond, JL, Shen, J: Velocity-correction projection methods for incompressible flows. SIAM J. Numer. Anal. 41 112-134 (2003)

16. Guermond, JL, Shen, J: A new class of truly consistent splitting schemes for incompressible flows. J. Comput. Phys. $192,262-276(2003)$

17. Shen, J, Yang, $X$ : Error estimates for finite element approximations of consistent splitting schemes for incompressible flows. Discrete Contin. Dyn. Syst., Ser. B 8, 663-676 (2007)

18. E, W, Liu, JG: Gauge method for viscous incompressible flows. Commun. Math. Sci. 1, 317-332 (2003)

19. Shen, J: On error estimates of the projection methods for the Navier-Stokes equations: first-order schemes. SIAM J. Numer. Anal. 29, 57-77 (1992)

20. Guermond, JL, Quartapelle, L: On the approximation of the unsteady Navier-Stokes equations by finite element projection methods. Numer. Math. 80, 207-238 (1998)

21. Guermond, JL, Quartapelle, L: Calculation of incompressible viscous flows by an unconditionally stable projection FEM. J. Comput. Phys. 132, 12-33 (1997)

22. Guermond, JL, Salgado, A: A splitting method for incompressible flows with variable density based on a pressure Poisson equation. J. Comput. Phys. 228, 2834-2846 (2009)

23. Nochetto, R, Pyo, J: The gauge-Uzawa finite element method. Part I: the Navier-Stokes equations. SIAM J. Numer. Anal. 43, 1043-1086 (2005)

24. Nochetto, R, Pyo, JH: The finite element gauge-Uzawa method. Part II: the Boussinesq equations. Math. Models Methods Appl. Sci. 16, 1599-1626 (2006)

25. Pyo, J, Shen, J: Gauge-Uzawa methods for incompressible flows with variable density. J. Comput. Phys. 221, 181-197 (2001)

26. Douglas, J Jr., Russell, TF: Numerical methods for convection-dominated diffusion problems based on combing the method of characteristics with finite element or finite difference procedures. SIAM J. Numer. Anal. 19, 871-885 (1982)

27. Russell, TF: Time stepping along characteristics with incomplete iteration for a Galerkin approximation of miscible displacement in porous media. SIAM J. Numer. Anal. 22, 970-1013 (1985)

28. Dawson, CN, Russell, TF, Wheeler, MF: Some improved error estimates for the modified method of characteristics. SIAM J. Numer. Anal. 26, 1487-1512 (1989) 
29. Buscaglia, G, Dari, EA: Implementation of the Lagrange-Galerkin method for the incompressible for the incompressible Navier-Stokes equations. Int. J. Numer. Methods Fluids 15, 23-36 (1992)

30. Si, ZY: Second order modified method of characteristics mixed defect-correction finite element method for time dependent Navier-Stokes problems. Numer. Algorithms 59, 271-300 (2012)

31. Notsu, H, Rui, H, Tabata, M: Development and L2-analysis of a single-step characteristics finite difference scheme of second order in time for convection-diffusion problems. J. Algorithms Comput. Technol. 7, 343-380 (2013)

32. Notsu, H, Tabata, M: A single-step characteristic-curve finite element scheme of second order in time for the incompressible Navier-Stokes equations. J. Sci. Comput. 38, 1-14 (2009)

33. El-Amrani, M, Seaid, M: Convergence and stability of finite element modified method of characteristics for the incompressible Navier-Stokes equations. J. Numer. Math. 15, 101-135 (2007)

34. El-Amrani, M, Seaid, M: Numerical simulation of natural and mixed convection flows by Galerkin-characteristic method. Int. J. Numer. Methods Fluids 53, 1819-1845 (2007)

35. Achdou, Y, Guermond, JL: Convergence analysis of a finite element projection/Lagrange-Galerkin method for the incompressible Navier-Stokes equations. SIAM J. Numer. Anal. 37, 799-826 (2000)

36. Boukir, K, Maday, Y, Métivet, B, Razafindrakoto, E: A higher-order characteristics/finite element method for the incompressible Navier-Stokes equations. Int. J. Numer. Methods Fluids 25, 1421-1454 (1997)

37. Si, Z, He, Y, Wang, Y: Modified characteristics mixed defect-correction finite element method for the time-dependent Navier-Stokes problems. Appl. Anal. 94, 701-724 (2015)

38. Si, ZY, Song, XG, Huang, PZ: Modified characteristics gauge-Uzawa finite element method for time dependent conduction-convection problems. J. Sci. Comput. 58, 1-24 (2014)

39. He, YN, Wang, AW: A simplified two-level method for the steady Navier-Stokes equations. Comput. Methods Appl. Mech. Eng. 197, 1568-1576 (2008)

40. Li, J, Chen, ZX: A new local stabilized non conforming finite element method for the Stokes equations. Computing 82, 157-170 (2008)

41. Temam, R: Navier-Stokes Equations: Theory and Numerical Analysis. North-Holland, Amsterdam (1984)

42. Hecht, F, Pironneau, O, Ohtsuka, K: FreeFEM++ (2010). http://www.freefem.org/ff++/

\section{Submit your manuscript to a SpringerOpen ${ }^{\ominus}$ journal and benefit from:}

- Convenient online submission

- Rigorous peer review

- Immediate publication on acceptance

Open access: articles freely available online

- High visibility within the field

- Retaining the copyright to your article 